\title{
THE CHAMBER GRAPH OF THE $M_{24}$ MAXIMAL 2-LOCAL GEOMETRY
}

\author{
PETER ROWLEY
}

\begin{abstract}
The chamber graph of the maximal 2-local geometry for $M_{24}$, the Mathieu group of degree 24, is analysed extensively. In addition to determining the discs around a fixed chamber of the chamber graph, the geodesic closure of an opposite pair of chambers is investigated.
\end{abstract}

\section{Introduction}

By a geometry (over the set $I$ ) we mean a triple $(\Gamma, \tau, *)$ where $\Gamma$ is a set, $\tau$ an onto map from $\Gamma$ to $I$ and $*$ is a symmetric relation on $\Gamma$ with the property that for $x, y \in \Gamma, x * y$ implies $\tau(x) \neq \tau(y)$. We refer to $*$ as the incidence relation and $\tau$ as the type map of the geometry; $x \in \Gamma$ is said to have type $i$ if $\tau(x)=i$. For brevity, we write $\Gamma$ to stand for $(\Gamma, \tau, *)$. A flag $F$ is a set of pairwise incident elements of $\Gamma$. The rank of $\Gamma$ is $|I|$ and the rank of $F$ is $|\{\tau(x) \mid x \in F\}|$. Let $\mathcal{C}$ denote the set of maximal flags (or chambers) of $\Gamma$ - a flag $F$ is maximal provided its rank is $|I|$. The chamber graph of $\Gamma$ has $\mathcal{C}$ as its vertex set and two (distinct) chambers $F_{1}$ and $F_{2}$ are adjacent whenever the rank of $F_{1} \cap F_{2}$ is $|I|-1$. We will denote the chamber graph of $\Gamma$ by $\mathcal{C}$.

For further discussion on chamber systems see [7, 9] and for background on geometries consult [1].

Here we will be solely concerned with elucidating the combinatorial structure and uncovering interesting properties of the chamber graph of the $M_{24}$ maximal 2-local geometry. This geometry first saw the light of day in [6], accompanied by the following diagram.

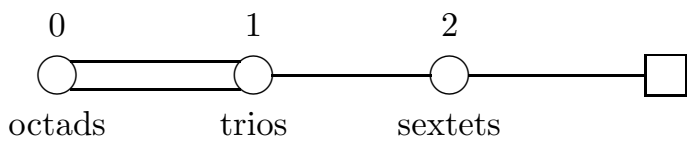

The square box is sometimes called a ghost node. There are no ghouls in this paper - we shall regard this as a rank 3-geometry, henceforth denoted $\Gamma$. The elements of $\Gamma$ are the octads, trios and sextets of the Steiner system $S(24,8,5)$, with octads, trios and sextets having, respectively, type 0,1 and 2 , where $I=\{0,1,2\}$. An octad is deemed to be incident with a trio if it is one of the three octads of the trio, and to be incident with a sextet if it may be obtained as the union of two of the tetrads of the sextet. A trio and a sextet are incident if the three octads of the trio are unions of the tetrads of the sextet. 
So, at a more down to earth level, an element (chamber) of $\mathcal{C}$ consists of an octad, trio and sextet such that the trio may be obtained from the sextet by a suitable pairing of the tetrads and the octad is one of the octads of the trio. Throughout this paper $G$ denotes the copy of $M_{24}$ preserving the Steiner system determined by Curtis's standard MOG array [4]. We recall that $G$ is a subgroup of Aut $\Gamma$ which acts transitively on the chambers of $\Gamma$ (that is the vertices of $\mathcal{C}$ ).

Apart from anything to do with $M_{24}$ being of intrinsic interest, it is the geometric context that motivates much of our investigations. In the geometric world $\Gamma$ occupies an exalted position by virtue of its appearance as a residue geometry in other geometries associated with $\cdot 1, M, F i_{24}$ and $J_{4}$ (see [6] again). The class of buildings is also much revered in the geometry world. In fact, the chamber graph of a building encodes many of the building axioms and concepts (such as galleries, thin subgeometries - see $[\mathbf{7}, \mathbf{8}]$ for more on this). This then leads us to compare and contrast the chamber graph of other geometries with those of buildings.

We use $d($,$) to denote the distance function on the chamber graph \mathcal{C}$ and, for $c \in \mathcal{C}$, define the $j$ th disc of $c$ to be

$$
D_{j}(c):=\left\{c^{\prime} \in \mathcal{C} \mid d\left(c, c^{\prime}\right)=j\right\} .
$$

Observing that $\Gamma$ has $1771 \cdot 15 \cdot 3=79,695$ chambers, we come to our first result.

THEOREM 1. Let $c_{0}$ be a fixed chamber in $\mathcal{C}$. Then the sizes of $D_{j}\left(c_{0}\right)$ are as follows.

\begin{tabular}{|c|c|c|c|c|c|c|c|c|c|c|c|}
\hline$j$ & 0 & 1 & 2 & 3 & 4 & 5 & 6 & 7 & 8 & 9 & 10 \\
\hline$\left|D_{j}\left(c_{0}\right)\right|$ & 1 & 10 & 44 & 184 & 544 & 1536 & 4800 & 10368 & 22272 & 38400 & 1536 \\
\hline
\end{tabular}

Theorem 1 is a consequence of a much more detailed picture of $\mathcal{C}$ which is unveiled in Section 3. Various other properties of $\mathcal{C}$ also emerge; concerning the last disc $D_{10}\left(c_{0}\right)$ we mention

THEOREM 2. Let $c_{0}$ be a fixed chamber in $\mathcal{C}$. Then $G_{c_{0}}$ is transitive on $D_{10}\left(c_{0}\right)$ and, furthermore, $D_{10}\left(c_{0}\right)$ is a coclique.

Theorem 2 leads us to view pairs of chambers which are distance 10 apart (in $\mathcal{C}$ ) with some interest. We shall call such a pair of chambers in $\mathcal{C}$ an opposite pair of chambers.

For $c, c^{\prime} \in \mathcal{C}$ a geodesic (or minimal gallery) from $c$ to $c^{\prime}$ is a shortest path in the graph $\mathcal{C}$, starting with $c$ and ending with $c^{\prime}$. Let $\mathcal{X}$ be a subset of $\mathcal{C}$. The geodesic closure of $\mathcal{X}$, denoted by $\overline{\mathcal{X}}$, consists of all the chambers in $\mathcal{C}$ which lie on a geodesic between $c$ and $c^{\prime}$ where $c, c^{\prime} \in \mathcal{X}$. The geodesic closure of two chambers that are maximal distance apart in the chamber graph of a building of spherical type yields (the chambers of) an apartment and every apartment of the building appears in this way (see $[6 ; 2.15$ Theorem and 3.8 Theorem]). This leads us to the subject of our next result.

THEOREM 3. Suppose $\left\{c_{0}, c_{10}\right\}$ is an opposite pair of chambers in $\mathcal{C}$, and set $\Delta_{j}=$ $\overline{\left\{c_{0}, c_{10}\right\}} \cap D_{j}\left(c_{0}\right)$. Then the sizes of $\Delta_{j}$ are as follows.

\begin{tabular}{|c|c|c|c|c|c|c|c|c|c|c|c|}
\hline$j$ & 0 & 1 & 2 & 3 & 4 & 5 & 6 & 7 & 8 & 9 & 10 \\
\hline$\left|\Delta_{j}\right|$ & 1 & 10 & 12 & 14 & 15 & 14 & 15 & 14 & 12 & 10 & 1 \\
\hline
\end{tabular}

In Section 4 we scrutinize $\overline{\left\{c_{0}, c_{10}\right\}}$ in some detail; indeed in an appendix we give a listing of all 118 chambers in $\overline{\left\{c_{0}, c_{10}\right\}}$ for a specific $c_{0}$ and $c_{10}$ since we 
believe/hope this set of chambers will repay further study. We note that the 'symmetry' of the sizes in Theorem 3 is a consequence of $G$ being transitive on $\mathcal{C}$ and $D_{10}\left(c_{0}\right)$ being a $G_{c_{0}}$-orbit. Further properties and a discussion of this graph may be found in $[8]$.

Briefly, this paper is arranged as follows. In the next section, we review properties of the Steiner system $S(24,8,5)$ with, as might be expected, particular emphasis on the octads, trios and sextets. We employ Curtis's MOG [4] both as our main descriptive device and also as an essential tool in our calculations. Accordingly, in Section 2, we spruce up MOG ready for action in Section 3 where we determine the $\operatorname{discs} D_{j}\left(c_{0}\right)$ for a fixed $c_{0} \in \mathcal{C}$. As mentioned above, Section 4 examines the geodesic closure of a pair of opposite chambers in $\mathcal{C}$, as well as airing some questions about $\mathcal{C}$, with further details given in Appendix A and Appendix B. Finally, Appendix $\mathrm{C}$ gives some MAGMA code (supplied by the referee who we thank) which calculates the lengths of the $G_{c_{0}}$-orbits on $\mathcal{C}$. This is to act as a check for some of the data in $(3.2)$.

\section{Notation and CHAMBERMOG}

Let $\Omega$ be a 24-element set which we assume comes equipped with the Steiner system $S(24,8,5)$. We denote the set of all octads, trios and sextets of $\Omega$ by, respectively, $\mathcal{O}, \mathcal{T}$ and $\mathcal{S}$.

For $c \in \mathcal{C}$, we use, respectively, $O(c), T(c)$ and $S(c)$ to denote the octad, trio and sextet of $c$. So $T(c)$ is obtained via a suitable partition of the tetrads of $S(c)$ and $O(c)$ is one of the three octads of $T(c)$. The three heavy blocks of the MOG are labelled thus

$$
\Omega=\begin{array}{|l|l|l|}
O_{1} & O_{2} & O_{3} \\
& & \\
\hline
\end{array}
$$

By the terms standard trio and standard sextet we mean the following trio and sextet.

$$
T_{0}:=\begin{array}{cc|cc|cc|}
+ & + & - & - & 0 & 0 \\
+ & + & - & - & \circ & \circ \\
+ & + & - & - & \circ & \circ \\
+ & + & - & - & \circ & \circ \\
\hline
\end{array}
$$$$
S_{0}:=\begin{array}{cc|cc|cc|}
+ & - & \times & * & \circ & \diamond \\
+ & - & \times & * & \circ & \diamond \\
+ & - & \times & * & \circ & \diamond \\
+ & - & \times & * & \circ & \diamond \\
\hline
\end{array}
$$

Throughout this paper, $c_{0}$ denotes the chamber of $\mathcal{C}$ for which $O\left(c_{0}\right)=O_{1}$, $T\left(c_{0}\right)=T_{0}$ and $S\left(c_{0}\right)=S_{0}$. Let $B$ denote the stabilizer in $G \cong M_{24}$ of $c_{0}$. Then $B=G_{O_{1}} \cap G_{T_{0}} \cap G_{S_{0}}$ has shape $2^{6}:\left(3 \times D_{8}\right): 2$ and $B=M N_{B}(\langle\rho\rangle)$ where

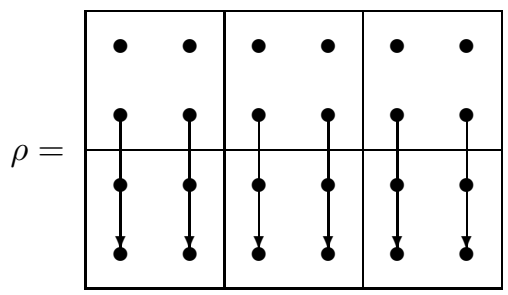


and $M=\left\langle g_{i} \mid 1 \leqslant i \leqslant 6\right\rangle$ and $N_{B}(\langle\rho\rangle)=\left\langle\rho, g_{7}, g_{8}, g_{9}, g_{10}\right\rangle$ with
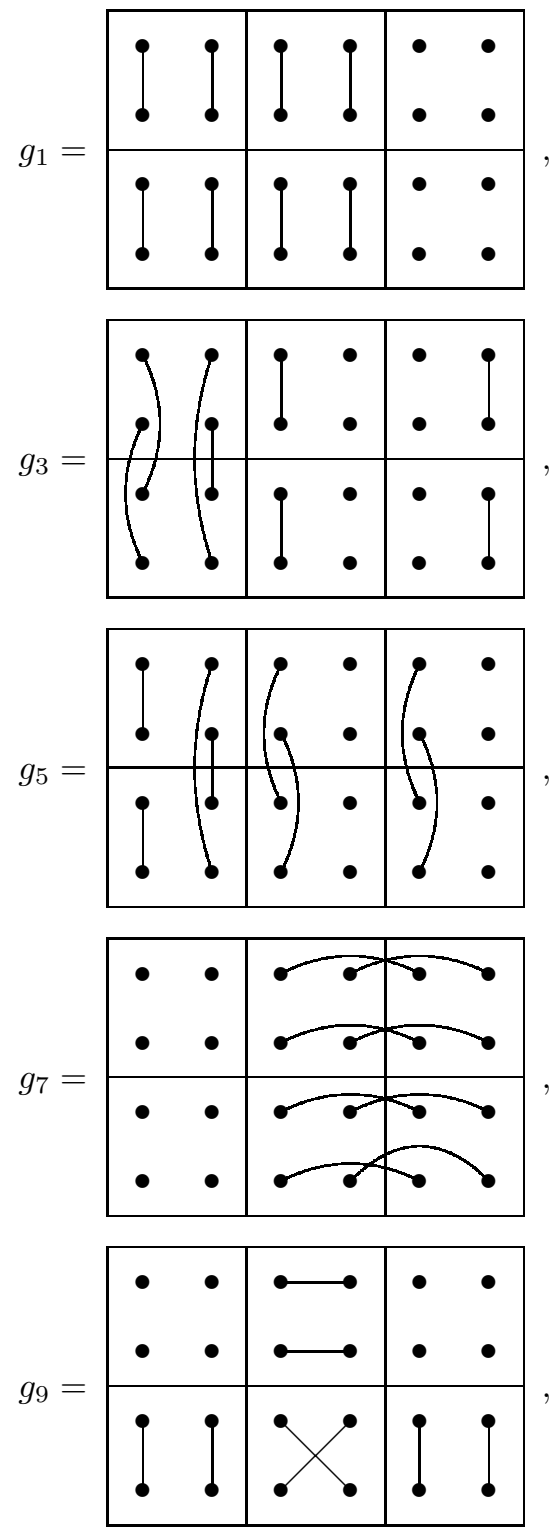
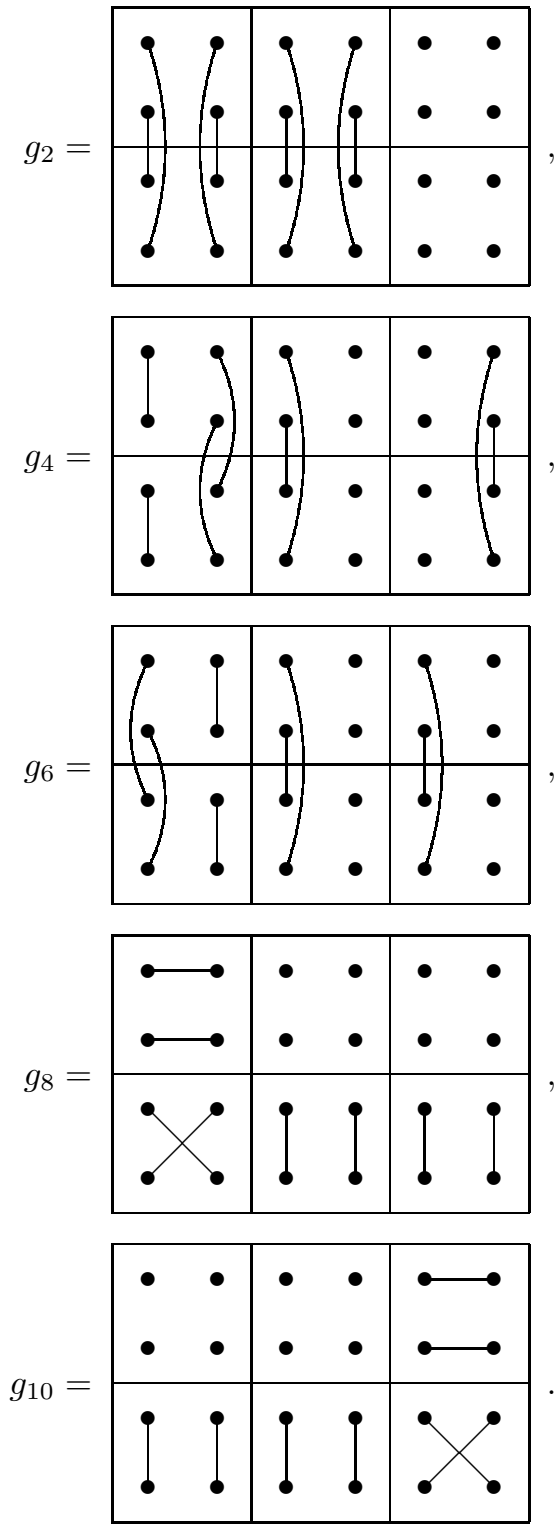

We note that $B=N_{G}(\langle\rho\rangle)$ is the stabilizer of the top row of the MOG and is isomorphic to the triple cover $3 \cdot S_{6}$. Further, $M$ is an elementary abelian group of order $2^{6}$ whose elements may also be labelled by the words of the hexacode. If $\{0,1, \omega, \bar{\omega}\}$ are the elements of the Galois field $G F(4)$, then 0 corresponds to the identity (on a column), 1 corresponds to interchanging the 1 st and 2 nd, 3rd and 4 th entries, $\omega$ corresponds to interchanging the 1st and 3rd, 2nd and 4th entries and $\bar{\omega}$ corresponds to interchanging the 1 st and 4 th, 2 nd and $3 r d$ entries. So $g_{4}$ corresponds to the hexacode word $(1, \omega, \bar{\omega}, 0,0, \bar{\omega})$. See the $M_{24}$ page in [2] for further details.

For distinct sextets $X_{1}$ and $X_{2}$, we have the following three possibilities for their 
intersection matrix. (The $(i, j)$ th entry is the number of elements in the intersection of the $i$ th tetrad of $X_{1}$ with the $j$ th tetrad of $X_{2}$, given a labelling of the tetrads of $X_{1}$ and $X_{2}$.)

$$
\begin{aligned}
& \mathcal{S}_{0}=\left(\begin{array}{llllll}
2 & & & & 1 & 1 \\
& 2 & & & 1 & 1 \\
& & 2 & & 1 & 1 \\
& & & 2 & 1 & 1 \\
1 & 1 & 1 & 1 & & \\
1 & 1 & 1 & 1 &
\end{array}\right) \quad \mathcal{S}_{1}=\left(\begin{array}{llllll}
3 & 1 & & & & \\
1 & 3 & & & & \\
& & 1 & 1 & 1 & 1 \\
& & 1 & 1 & 1 & 1 \\
& & 1 & 1 & 1 \\
& & 1 & 1 & 1 & 1
\end{array}\right) \\
& \mathcal{S}_{3}=\left(\begin{array}{llllll}
2 & 2 & & & \\
2 & 2 & & & \\
& & 2 & 2 & \\
& 2 & 2 & & \\
& & & & 2 & 2 \\
& & & 2 & 2
\end{array}\right)
\end{aligned}
$$

For a fixed sextet $X$ let $\sigma_{i}(X)$ denote the set of sextets which have an $\mathcal{S}_{i}$ intersection matrix with $X, i=0,1,3$. (So $\mathcal{S}=\{X\} \cup \sigma_{0}(X) \cup \sigma_{1}(X) \cup \sigma_{3}(X)$.) When $X=S_{0}$ we will write $\sigma_{i}$ in place of $\sigma_{i}(X)$. Note that for $X_{1} \in \sigma_{i}(X), X_{1}$ and $X$ have precisely $i$ octads in common.

(2.1) Let $X$ be a fixed sextet. Then

(i) $\left|\sigma_{0}(X)\right|=1440,\left|\sigma_{1}(X)\right|=240,\left|\sigma_{3}(X)\right|=90$; and

(ii) the $G_{X}$-orbits on the set of sextets are $X, \sigma_{0}(X), \sigma_{1}(X), \sigma_{3}(X)$.

Proof. See [3, Chapter 1, Section 2].

Next we consider trios. Let $Y_{1}$ and $Y_{2}$ be distinct trios. Then there are four possible ways their octads can intersect. Here the $(i, j)$ th entry is the size of the intersection of the $i$ th octad of $Y_{1}$ with the $j$ th octad of $Y_{2}$, assuming a labelling of the octads of $Y_{1}$ and $Y_{2}$.

$$
\begin{array}{ll}
\mathcal{T}_{0}=\left(\begin{array}{lll}
4 & 2 & 2 \\
2 & 4 & 2 \\
2 & 2 & 4
\end{array}\right) & \mathcal{T}_{1}=\left(\begin{array}{lll}
2 & 2 & 4 \\
2 & 2 & 4 \\
4 & 4 & 0
\end{array}\right) \\
\mathcal{T}_{2}=\left(\begin{array}{lll}
8 & 0 & 0 \\
0 & 4 & 4 \\
0 & 4 & 4
\end{array}\right) & \mathcal{T}_{3}=\left(\begin{array}{lll}
4 & 4 & 0 \\
4 & 0 & 4 \\
0 & 4 & 4
\end{array}\right)
\end{array}
$$

For a trio $Y, \tau_{i}(Y)$ will denote the set of trios whose octad intersection matrix with $Y$ is $\mathcal{T}_{i}, i=0,1,2,3$. In the case when $Y=T_{0}$, we shorten $\tau_{i}(Y)$ to $\tau_{i}$.

We usually describe a sextet $X$ in the following manner. For $i \in\{1, \ldots, 6\}$ the 4 -element subset of the MOG labelled by $i$ defines the tetrads of $X$. So

$$
S_{0}:=\begin{array}{ll|ll|ll|}
1 & 2 & 3 & 4 & 5 & 6 \\
1 & 2 & 3 & 4 & 5 & 6 \\
1 & 2 & 3 & 4 & 5 & 6 \\
1 & 2 & 3 & 4 & 5 & 6 \\
\hline
\end{array} .
$$


Also note that, for example,

\begin{tabular}{|ll|ll|ll|}
\hline 2 & 1 & 3 & 3 & 3 & 3 \\
1 & 2 & 4 & 4 & 4 & 4 \\
1 & 2 & 5 & 5 & 5 & 5 \\
1 & 2 & 6 & 6 & 6 & 6 \\
\hline
\end{tabular}

\begin{tabular}{|ll|ll|ll|}
\hline 5 & 4 & 1 & 1 & 1 & 1 \\
4 & 5 & 2 & 2 & 2 & 2 \\
4 & 5 & 3 & 3 & 3 & 3 \\
4 & 5 & 6 & 6 & 6 & 6 \\
\hline
\end{tabular}

describe the same sextet, say $X_{1}$.

We employ two schemes for describing chambers which we explain with an example. By

$$
\left.c_{1}:=\begin{array}{ll|ll|ll}
2 & 1 & 3 & 3 & 3 & 3 \\
1 & 2 & 4 & 4 & 4 & 4 \\
1 & 2 & 5 & 5 & 5 & 5 \\
1 & 2 & 6 & 6 & 6 & 6
\end{array}\right] \quad \text { or } \quad c_{1}:=\begin{array}{lll|ll|ll|}
2 & 1 & 3^{+} & 3 & 3 & 3 \\
1 & 2 & 4^{+} & 4 & 4 & 4 \\
1 & 2 & 5^{-} & 5 & 5 & 5 \\
1 & 2 & 6^{-} & 6 & 6 & 6 \\
\hline
\end{array}
$$

$\underline{12}|34| 56$

we mean that $c_{1}$ is a chamber with $O\left(c_{1}\right)=O_{1}, T\left(c_{1}\right)=T_{0}$ and $S\left(c_{1}\right)=X_{1}$. The $\underline{12}|34| 56$ tells us how to partition the tetrads to obtain the trio and the underlined partition gives the octad of the chamber. In the alternative notation the + on the 3 and 4 and the - on the 5 and 6 indicate two of the octads of the trio and the 'unmarked' octad is the octad of the chamber. Dressed in this way MOG becomes CHAMBERMOG.

\section{B-orbits and disc structure}

Our strategy for determining the chambers in a particular $D_{j}\left(c_{0}\right)$ is first to determine the $B$-orbits of $\mathcal{C}$. Clearly, if a chamber $c$ is in $D_{j}\left(c_{0}\right)$, then the $B$-orbit of $c$ will be contained in $D_{j}\left(c_{0}\right)$. Beginning with $c_{0}$ we work outwards building up successive discs of $c_{0}$. Suppose we have successfully enumerated $D_{i}\left(c_{0}\right)$ for $i \leqslant j$. Taking $c \in D_{j}\left(c_{0}\right)$ we calculate, using the MOG, the ten chambers of $\mathcal{C}$ adjacent to $c$. In the light of our knowledge of $D_{i}\left(c_{0}\right), i \leqslant j$, we discover which of the ten chambers are in $D_{j+1}\left(c_{0}\right)$, and hence the $B$-orbits of these chambers will be in $D_{j+1}\left(c_{0}\right)$. Letting $c$ run through representatives of the $B$-orbits of $D_{j}\left(c_{0}\right)$, this procedure delivers $D_{j+1}\left(c_{0}\right)$ as a union of $B$-orbits. And we repeat this procedure until we run out of chambers!

We arrive at the orbits of $B$ on the chambers of $\mathcal{C}$ in a series of stages. First we determine the orbits of $B$ upon the sextets of $\Omega$. Then taking a representative sextet $X$ from each of these we may obtain the 15 trios incident with $X$ whence, by nominating a particular octad, we then get the 45 chambers which contain $X$. Examining the action of $B_{X}$ and combinatorial properties of such chambers in relation to $c_{0}$ allows us to compile the $B$-orbits of $\mathcal{C}$.

In order to describe the $B$-orbits of $\mathcal{S}$ we need some auxiliary notions.

Let $X \in \mathcal{S}$, and first we suppose that $X \in \sigma_{0}$. Then there will be precisely two columns (of the MOG) and four tetrads of $X$ each of which intersect the two columns (of the MOG) in one element. We shall refer to either of these two columns 
of the MOG as mixed cols of $X$. So, for example, if

$$
X=\begin{array}{ll|ll|ll}
6 & 4 & 3 & 2 & 5 & 4 \\
3 & 3 & 5 & 1 & 5 & 6 \\
5 & 1 & 2 & 2 & 1 & 6 \\
2 & 3 & 6 & 4 & 4 & 1 \\
\hline
\end{array}
$$

then the first and third columns of the MOG are the mixed cols of $X$. Now consider the case when $X \in \sigma_{1}$. Here we have exactly two columns of the MOG for which two of the tetrads of $X$ intersect these columns in 3 elements. Such columns of the MOG will be called 3-cols of $X$. If, say,

$$
X=\begin{array}{ll|ll|ll|}
3 & 3 & 3 & 3 & 1 & 2 \\
4 & 4 & 4 & 4 & 2 & 1 \\
5 & 5 & 5 & 5 & 2 & 1 \\
6 & 6 & 6 & 6 & 2 & 1 \\
\hline
\end{array}
$$

then the fifth and sixth columns of the MOG are the 3-cols of $X$. Lastly we look at $X \in \sigma_{3}$. In this case the six columns of the MOG are partitioned into three pairs where each pair of columns is determined by two tetrads of $X$ each of which intersects both columns of the pair in two elements. Any three of these pairs of columns we call a col pair of $X$. If, for example,

$$
X=\begin{array}{ll|ll|ll}
5 & 6 & 2 & 3 & 4 & 1 \\
6 & 5 & 2 & 3 & 4 & 1 \\
6 & 6 & 4 & 1 & 2 & 3 \\
5 & 5 & 4 & 1 & 2 & 3 \\
\hline
\end{array}
$$

then the col pairs of $X$ are columns 1 and 2, columns 3 and 5, and columns 4 and 6 .

Since $B \leqslant G_{S_{0}}$, we see that $\sigma_{i}(i=0,1,3)$ will be a union of $B$-orbits. Hence we label the $B$-orbits of $\mathcal{S}$ by $\sigma_{i}^{(n)}$ to indicate that $\sigma_{i}^{(n)} \subseteq \sigma_{i}(i=0,1,3)$; the $n$ is the size of that particular $B$-orbit.

(3.1) $B$ has 12-orbits on $\mathcal{S}$ the details of which are tabulated below.

\begin{tabular}{|c|c|l|}
\hline$B$-ORBIT & SIZE & \multicolumn{1}{|c|}{ DESCRIPTION } \\
\hline$\left\{S_{0}\right\}$ & 1 & standard sextet \\
\hline$\sigma_{0}^{(96)}$ & 96 & both mixed cols in $O_{1}$ \\
\hline$\sigma_{0}^{(192)}$ & 192 & both mixed cols either in $O_{2}$ or in $O_{3}$ \\
\hline$\sigma_{0}^{(384)}$ & 384 & one mixed col in $O_{2}$, one mixed col in $O_{3}$ \\
\hline$\sigma_{0}^{(768)}$ & 768 & one mixed col in $O_{1}$, one mixed col either in $O_{2}$ or in $O_{3}$ \\
\hline$\sigma_{1}^{(16)}$ & 16 & both 3-cols in $O_{1}$ \\
\hline$\sigma_{1}^{(32)}$ & 32 & both 3-cols either in $O_{2}$ or in $O_{3}$ \\
\hline$\sigma_{1}^{(64)}$ & 64 & one 3-col in $O_{2}$, one 3-col in $O_{3}$ \\
\hline$\sigma_{1}^{(128)}$ & 128 & one 3-col in $O_{1}$, one 3-col either in $O_{2}$ or in $O_{3}$ \\
\hline$\sigma_{3}^{(6)}$ & 6 & each col pair contained in one of $O_{1}, O_{2}, O_{3}$ \\
\hline$\sigma_{3}^{(12)}$ & 12 & one col pair in $O_{1}$, no col pairs either in $O_{2}$ or in $O_{3}$ \\
\hline$\sigma_{3}^{(24)}$ & 24 & $\begin{array}{l}\text { one col pair in } O_{2}, \text { no col pairs either in } O_{1} \text { or in } O_{3} \text { or } \\
\text { one col pair in } O_{3}, \text { no col pair either in } O_{1} \text { or in } O_{2}\end{array}$ \\
\hline$\sigma_{3}^{(48)}$ & 48 & no col pairs in any of $O_{1}, O_{2}, O_{3}$ \\
\hline
\end{tabular}


Building upon the information in (3.1) we obtain the $B$-orbits of $\mathcal{C}$ and then we implement the procedure discussed earlier, the results of which, together with the $B$-orbits of $\mathcal{C}$, are presented in (3.2). After (3.2) we explain how the MOG helps us to calculate the adjacent chambers of a given chamber. In columns $2,3,4$ and 5 of the tables in (3.2) we give, respectively, the sizes of a $B$-orbit, the $j$ for which $D_{j}\left(c_{0}\right)$ contains the $B$-orbit, $\tau_{i}$ for which $T(c) \in \tau_{i}$ and $\left|O(c) \cap O_{1}\right|$ (where $c$ is a chamber in the $B$-orbit).

$S(c)=S_{0}:$

$S(c)=$\begin{tabular}{ll|ll|ll|}
1 & 2 & 3 & 4 & 5 & 6 \\
1 & 2 & 3 & 4 & 5 & 6 \\
1 & 2 & 3 & 4 & 5 & 6 \\
1 & 2 & 3 & 4 & 5 & 6 \\
\hline
\end{tabular},

\begin{tabular}{|c|c|c|c|c|}
\hline $\begin{array}{l}B \text {-orbit } \\
\text { representative }\end{array}$ & size & disc & $\tau_{i}$ & $\begin{array}{l}O_{1-} \\
\text { intersection }\end{array}$ \\
\hline$\underline{12}|34| 56$ & 1 & 0 & $\tau_{0}$ & 8 \\
\hline $12|\underline{34}| 56$ & 2 & 1 & $\tau_{0}$ & 0 \\
\hline$\underline{12}|35| 46$ & 2 & 1 & $\tau_{2}$ & 8 \\
\hline $15|26| \underline{34}$ & 4 & 2 & $\tau_{2}$ & 0 \\
\hline $12|\underline{35}| 46$ & 4 & 2 & $\tau_{2}$ & 0 \\
\hline$\underline{15}|26| 34$ & 8 & 3 & $\tau_{2}$ & 4 \\
\hline $14|26| \underline{35}$ & 8 & 3 & $\tau_{3}$ & 0 \\
\hline$\underline{13}|25| 46$ & 16 & 4 & $\tau_{3}$ & 4 \\
\hline
\end{tabular}

$S(c) \in \sigma_{0}^{(96)}:$

$S(c)=$\begin{tabular}{|ll|ll|ll|}
\hline 1 & 3 & 5 & 1 & 3 & 5 \\
2 & 4 & 6 & 1 & 3 & 6 \\
3 & 2 & 2 & 5 & 5 & 4 \\
4 & 1 & 2 & 6 & 6 & 4 \\
\hline
\end{tabular}

\begin{tabular}{|c|c|c|c|c|}
\hline $\begin{array}{l}B \text {-orbit } \\
\text { representative }\end{array}$ & size & disc & $\tau_{i}$ & $\begin{array}{l}O_{1^{-}} \\
\text {interesetion }\end{array}$ \\
\hline $12|34| \underline{56}$ & $1 \times 96$ & 5 & $\tau_{3}$ & 0 \\
$13|24| \underline{56}$ & $2 \times 96$ & 6 & $\tau_{1}$ & 0 \\
$\underline{12}|34| 56$ & $2 \times 96$ & 6 & $\tau_{3}$ & 4 \\
$\underline{13}|24| 56$ & $4 \times 96$ & 7 & $\tau_{1}$ & 4 \\
$\underline{12}|35| 46$ & $4 \times 96$ & 7 & $\tau_{1}$ & 4 \\
$\underline{13}|25| 46$ & $8 \times 96$ & 8 & $\tau_{0}$ & 4 \\
$\underline{15}|26| 34$ & $8 \times 96$ & 8 & $\tau_{1}$ & 2 \\
$\underline{15}|23| 46$ & $16 \times 96$ & 9 & $\tau_{0}$ & 2 \\
\hline
\end{tabular}

$S(c) \in \sigma_{0}^{(192)}:$

$S(c)=$\begin{tabular}{ll|ll|ll|}
5 & 1 & 1 & 3 & 3 & 5 \\
6 & 1 & 2 & 4 & 3 & 6 \\
2 & 5 & 3 & 2 & 5 & 4 \\
2 & 6 & 4 & 1 & 6 & 4 \\
\hline
\end{tabular},

\begin{tabular}{|c|c|c|c|c|}
\hline $\begin{array}{l}B \text {-orbit } \\
\text { representative }\end{array}$ & size & disc & $\tau_{i}$ & $\begin{array}{l}O_{1-} \\
\text { intersection }\end{array}$ \\
\hline $12|\underline{34}| 56$ & $1 \times 192$ & 5 & $\tau_{3}$ & 0 \\
\hline $15|\underline{34}| 26$ & $2 \times 192$ & 6 & $\tau_{1}$ & 0 \\
\hline $12|34| \underline{56}$ & $1 \times 192$ & 6 & $\tau_{3}$ & 4 \\
\hline$\underline{12}|34| 56$ & $1 \times 192$ & 6 & $\tau_{3}$ & 4 \\
\hline$\underline{16}|25| 34$ & $4 \times 192$ & 7 & $\tau_{1}$ & 4 \\
\hline$\underline{12}|35| 46$ & $2 \times 192$ & 7 & $\tau_{1}$ & 4 \\
\hline $13|24| \underline{56}$ & $2 \times 192$ & 7 & $\tau_{1}$ & 4 \\
\hline$\underline{15}|23| 46$ & $8 \times 192$ & 8 & $\tau_{0}$ & 4 \\
\hline $12|\underline{35}| 46$ & $4 \times 192$ & 8 & $\tau_{1}$ & 2 \\
\hline$\underline{13}|24| 56$ & $4 \times 192$ & 8 & $\tau_{1}$ & 2 \\
\hline $14|26| \underline{35}$ & $8 \times 192$ & 9 & $\tau_{0}$ & 2 \\
\hline$\underline{\underline{13}}|25| 46$ & $8 \times 192$ & 9 & $\tau_{0}$ & 2 \\
\hline
\end{tabular}


The chamber graph of the $M_{24}$ maximal 2-local geometry $S(c) \in \sigma_{0}^{(384)}:$

$S(c)=$\begin{tabular}{|ll|ll|ll|}
5 & 3 & 1 & 6 & 3 & 1 \\
4 & 5 & 2 & 5 & 4 & 1 \\
4 & 3 & 3 & 2 & 2 & 6 \\
6 & 6 & 4 & 2 & 1 & 5 \\
\hline
\end{tabular}

\begin{tabular}{|c|c|c|c|c|}
\hline $\begin{array}{l}B \text {-orbit } \\
\text { representative }\end{array}$ & size & disc & $\tau_{i}$ & $\begin{array}{l}O_{1-} \\
\text { intersection }\end{array}$ \\
\hline$\underline{12}|34| 56$ & $1 \times 384$ & 6 & $\tau_{1}$ & 0 \\
$\underline{12}|35| 46$ & $2 \times 384$ & 6 & $\tau_{1}$ & 0 \\
$12|\underline{35}| 46$ & $4 \times 384$ & 7 & $\tau_{1}$ & 4 \\
$12|\underline{34}| 56$ & $1 \times 384$ & 7 & $\tau_{1}$ & 4 \\
$12|34| \underline{56}$ & $1 \times 384$ & 7 & $\tau_{1}$ & 4 \\
$14|\underline{35}| 26$ & $8 \times 384$ & 8 & $\tau_{0}$ & 4 \\
$15|26| 34$ & $2 \times 384$ & 8 & $\tau_{0}$ & 4 \\
$14|\underline{56}| 23$ & $2 \times 384$ & 8 & $\tau_{0}$ & 4 \\
$\underline{13}|25| 46$ & $8 \times 384$ & 9 & $\tau_{0}$ & 2 \\
$14|\underline{25}| 36$ & $8 \times 384$ & 9 & $\tau_{0}$ & 2 \\
$\underline{16}|25| 34$ & $4 \times 384$ & 9 & $\tau_{0}$ & 2 \\
$14|\underline{23}| 56$ & $4 \times 384$ & 9 & $\tau_{0}$ & 2 \\
\hline
\end{tabular}

$S(c) \in \sigma_{0}^{(768)}:$

$S(c)=$\begin{tabular}{ll|ll|ll|}
6 & 4 & 3 & 2 & 5 & 4 \\
3 & 3 & 5 & 1 & 5 & 6 \\
5 & 1 & 2 & 2 & 1 & 6 \\
2 & 3 & 6 & 4 & 4 & 1 \\
\hline
\end{tabular}

\begin{tabular}{|c|c|c|c|c|}
\hline $\begin{array}{l}B \text {-orbit } \\
\text { representative }\end{array}$ & size & disc & $\tau_{i}$ & $\begin{array}{l}O_{1^{-}} \\
\text {intersection }\end{array}$ \\
\hline $14|\underline{23}| 56$ & $1 \times 768$ & 7 & $\tau_{1}$ & 4 \\
$15|\underline{23}| 46$ & $2 \times 768$ & 7 & $\tau_{1}$ & 4 \\
$\underline{14}|23| 56$ & $1 \times 768$ & 8 & $\tau_{1}$ & 2 \\
$14|23| \underline{56}$ & $1 \times 768$ & 8 & $\tau_{1}$ & 2 \\
$\underline{15}|23| 46$ & $4 \times 768$ & 8 & $\tau_{1}$ & 2 \\
$12|\underline{35}| 46$ & $4 \times 768$ & 8 & $\tau_{0}$ & 4 \\
$14 \mid 26$ & $\mid 35$ \\
$\underline{13}|24| 56$ & $2 \times 768$ & 8 & $\tau_{0}$ & 4 \\
$\underline{13}|25| 46$ & $4 \times 768$ & 9 & $\tau_{0}$ & 4 \\
$\underline{14}|25| 36$ & $2 \times 768$ & 9 & $\tau_{0}$ & 4 \\
$12|34| \underline{56}$ & $2 \times 768$ & 9 & $\tau_{0}$ & 2 \\
$12|36| \underline{45}$ & $4 \times 768$ & 9 & $\tau_{0}$ & 2 \\
$13|26| \underline{45}$ & $4 \times 768$ & 9 & $\tau_{0}$ & 2 \\
$\underline{12}|36| 45$ & $4 \times 768$ & 9 & $\tau_{0}$ & 2 \\
$14|\underline{25}| 36$ & $2 \times 768$ & 9 & $\tau_{0}$ & 2 \\
$13|\underline{25}| 46$ & $4 \times 768$ & 9 & $\tau_{0}$ & 2 \\
$\underline{12}|34| 56$ & $2 \times 768$ & 10 & $\tau_{0}$ & 2 \\
\hline
\end{tabular}

$S(c) \in \sigma_{1}^{(16)}:$

$S(c)=$\begin{tabular}{ll|ll|ll}
2 & 1 & 3 & 3 & 3 & 3 \\
1 & 2 & 4 & 4 & 4 & 4 \\
1 & 2 & 5 & 5 & 5 & 5 \\
1 & 2 & 6 & 6 & 6 & 6 \\
\hline
\end{tabular}

\begin{tabular}{|c|c|c|c|c|}
\hline $\begin{array}{l}B \text {-orbit } \\
\text { representative }\end{array}$ & size & disc & $\tau_{i}$ & $\begin{array}{l}O_{1^{-}} \\
\text {intersection }\end{array}$ \\
\hline$\underline{12}|34| 56$ & $3 \times 16$ & 3 & $\tau_{2}$ & 8 \\
\hline $12|\underline{34}| 56$ & $6 \times 16$ & 4 & $\tau_{2}$ & 0 \\
\hline $15|26| \underline{34}$ & $12 \times 16$ & 5 & $\tau_{1}$ & 0 \\
\hline$\underline{13}|24| 56$ & $24 \times 16$ & 6 & $\tau_{1}$ & 4 \\
\hline
\end{tabular}


The chamber graph of the $M_{24}$ maximal 2-local geometry

$S(c) \in \sigma_{1}^{(32)}$ :

$S(c)=$\begin{tabular}{|ll|ll|ll}
3 & 3 & 2 & 1 & 3 & 3 \\
4 & 4 & 1 & 2 & 4 & 4 \\
5 & 5 & 1 & 2 & 5 & 5 \\
6 & 6 & 1 & 2 & 6 & 6 \\
\hline
\end{tabular}

\begin{tabular}{|c|c|c|c|c|}
\hline $\begin{array}{l}B \text {-orbit } \\
\text { representative }\end{array}$ & size & disc & $\tau_{i}$ & $\begin{array}{l}O_{1^{-}} \\
\text {intersection }\end{array}$ \\
\hline$\underline{12}|34| 56$ & $3 \times 32$ & 4 & $\tau_{2}$ & 0 \\
$12|\underline{34}| 56$ & $6 \times 32$ & 5 & $\tau_{2}$ & 4 \\
$13|24| \underline{56}$ & $12 \times 32$ & 6 & $\tau_{1}$ & 4 \\
$\underline{13}|24| 56$ & $24 \times 32$ & 7 & $\tau_{1}$ & 2 \\
\hline
\end{tabular}

$S(c) \in \sigma_{1}^{(64)}:$

$S(c)=$\begin{tabular}{ll|ll|ll}
5 & 4 & 1 & 3 & 1 & 6 \\
6 & 5 & 2 & 5 & 1 & 4 \\
4 & 6 & 2 & 4 & 1 & 5 \\
3 & 3 & 2 & 6 & 2 & 3 \\
\hline
\end{tabular},

\begin{tabular}{|c|c|c|c|c|}
\hline $\begin{array}{l}B \text {-orbit } \\
\text { representative }\end{array}$ & size & disc & $\tau_{i}$ & $\begin{array}{l}O_{1^{-}} \\
\text {intersection }\end{array}$ \\
\hline$\underline{12}|34| 56$ & $3 \times 64$ & 5 & $\tau_{1}$ & 0 \\
$12|34| 56$ & $6 \times 64$ & 6 & $\tau_{1}$ & 4 \\
$13|24| 56$ & $12 \times 64$ & 7 & $\tau_{0}$ & 4 \\
$\underline{13}|24| 56$ & $24 \times 64$ & 8 & $\tau_{0}$ & 2 \\
\hline
\end{tabular}

$S(c) \in \sigma_{1}^{(128)}$ :

$S(c)=$\begin{tabular}{ll|ll|ll|}
1 & 4 & 5 & 3 & 1 & 6 \\
2 & 5 & 6 & 5 & 1 & 4 \\
2 & 6 & 4 & 4 & 1 & 5 \\
2 & 3 & 3 & 6 & 2 & 3 \\
\hline
\end{tabular}

\begin{tabular}{|c|c|c|c|c|}
\hline $\begin{array}{l}B \text {-orbit } \\
\text { representative }\end{array}$ & size & disc & $\tau_{i}$ & $\begin{array}{l}O_{1^{-}} \\
\text {intersection }\end{array}$ \\
\hline$\underline{12}|34| 56$ & $3 \times 128$ & 6 & $\tau_{1}$ & 4 \\
$12|\underline{34}| 56$ & $6 \times 128$ & 7 & $\tau_{1}$ & 2 \\
$13|24| \underline{56}$ & $12 \times 128$ & 8 & $\tau_{0}$ & 2 \\
$\underline{13}|24| 56$ & $12 \times 128$ & 9 & $\tau_{0}$ & 2 \\
$14|\underline{23}| 56$ & $12 \times 128$ & 9 & $\tau_{0}$ & 4 \\
\hline
\end{tabular}

$S(c) \in \sigma_{3}^{(6)}:$

$S(c)=$\begin{tabular}{ll|ll|ll}
1 & 1 & 3 & 3 & 5 & 5 \\
1 & 1 & 3 & 3 & 5 & 5 \\
2 & 2 & 4 & 4 & 6 & 6 \\
2 & 2 & 4 & 4 & 6 & 6 \\
\hline
\end{tabular},

\begin{tabular}{|c|c|c|c|c|}
\hline $\begin{array}{l}B \text {-orbit } \\
\text { representative }\end{array}$ & size & disc & $\tau_{i}$ & $\begin{array}{l}O_{1^{-}} \\
\text {intersection }\end{array}$ \\
\hline$\underline{12}|34| 56$ & $1 \times 6$ & 1 & $\tau_{0}$ & 8 \\
$12|\underline{34}| 56$ & $2 \times 6$ & 2 & $\tau_{0}$ & 0 \\
$\underline{12}|35| 46$ & $2 \times 6$ & 2 & $\tau_{2}$ & 8 \\
$12|\underline{35}| 46$ & $4 \times 6$ & 3 & $\tau_{2}$ & 0 \\
$13|24| \underline{56}$ & $4 \times 6$ & 3 & $\tau_{2}$ & 0 \\
$13|26| \underline{45}$ & $8 \times 6$ & 4 & $\tau_{3}$ & 0 \\
$\underline{13}|24| 56$ & $8 \times 6$ & 4 & $\tau_{2}$ & 4 \\
$\underline{13}|25| 46$ & $16 \times 6$ & 5 & $\tau_{3}$ & 4 \\
\hline
\end{tabular}


The chamber graph of the $M_{24}$ maximal 2-local geometry

$S(c) \in \sigma_{3}^{(12)}:$

$S(c)=$\begin{tabular}{|ll|ll|ll|}
2 & 1 & 3 & 5 & 6 & 4 \\
1 & 2 & 3 & 5 & 6 & 4 \\
1 & 1 & 6 & 4 & 3 & 5 \\
2 & 2 & 6 & 4 & 3 & 5 \\
\hline
\end{tabular}

\begin{tabular}{|c|c|c|c|c|}
\hline $\begin{array}{l}B \text {-orbit } \\
\text { representative }\end{array}$ & size & disc & $\tau_{i}$ & $\begin{array}{l}O_{1-} \\
\text { intersection }\end{array}$ \\
\hline$\underline{12}|36| 45$ & $1 \times 12$ & 2 & $\tau_{2}$ & 8 \\
$\underline{12}|34| 56$ & $2 \times 12$ & 3 & $\tau_{2}$ & 8 \\
$\underline{12}|\underline{36}| 45$ & $2 \times 12$ & 3 & $\tau_{2}$ & 0 \\
$12|\underline{34}| 56$ & $4 \times 12$ & 4 & $\tau_{2}$ & 0 \\
$14|25| \underline{36}$ & $4 \times 12$ & 4 & $\tau_{1}$ & 0 \\
$15|26| \underline{34}$ & $8 \times 12$ & 5 & $\tau_{1}$ & 0 \\
$\underline{14}|25| 36$ & $8 \times 12$ & 5 & $\tau_{1}$ & 4 \\
$\underline{14}|23| 56$ & $16 \times 12$ & 6 & $\tau_{1}$ & 4 \\
\hline
\end{tabular}

$S(c) \in \sigma_{3}^{(24)}$ :

$S(c)=$\begin{tabular}{ll|ll|ll|}
1 & 3 & 1 & 3 & 5 & 5 \\
1 & 3 & 1 & 3 & 6 & 6 \\
2 & 4 & 2 & 4 & 6 & 5 \\
2 & 4 & 2 & 4 & 5 & 6 \\
\hline
\end{tabular},

\begin{tabular}{|c|c|c|c|c|}
\hline $\begin{array}{l}B \text {-orbit } \\
\text { representative }\end{array}$ & size & disc & $\tau_{i}$ & $\begin{array}{l}O_{1^{-}} \\
\text {intersection }\end{array}$ \\
\hline $12|34| \underline{56}$ & $1 \times 24$ & 3 & $\tau_{2}$ & 0 \\
$13|24| \underline{56}$ & $2 \times 24$ & 4 & $\tau_{2}$ & 0 \\
$\underline{12}|34| 56$ & $2 \times 24$ & 4 & $\tau_{2}$ & 4 \\
$\underline{13}|24| 56$ & $4 \times 24$ & 5 & $\tau_{2}$ & 4 \\
$\underline{12}|35| 46$ & $4 \times 24$ & 5 & $\tau_{1}$ & 4 \\
$\underline{13}|25| 46$ & $8 \times 24$ & 6 & $\tau_{1}$ & 4 \\
$\underline{15}|26| 34$ & $8 \times 24$ & 6 & $\tau_{1}$ & 2 \\
$\underline{15}|23| 46$ & $16 \times 24$ & 7 & $\tau_{1}$ & 2 \\
\hline
\end{tabular}

$S(c) \in \sigma_{3}^{(48)}:$

$S(c)=$\begin{tabular}{ll|ll|ll|}
1 & 4 & 2 & 6 & 3 & 5 \\
2 & 3 & 2 & 5 & 3 & 6 \\
2 & 4 & 1 & 6 & 4 & 6 \\
1 & 3 & 1 & 5 & 4 & 5 \\
\hline
\end{tabular}

\begin{tabular}{|c|c|c|c|c|}
\hline $\begin{array}{l}B \text {-orbit } \\
\text { representative }\end{array}$ & size & disc & $\tau_{i}$ & $\begin{array}{l}O_{1-} \\
\text { intersection }\end{array}$ \\
\hline $12|34| \underline{56}$ & $1 \times 48$ & 4 & $\tau_{3}$ & 0 \\
$13|24| \underline{56}$ & $2 \times 48$ & 5 & $\tau_{1}$ & 0 \\
$\underline{12}|34| 56$ & $2 \times 48$ & 5 & $\tau_{3}$ & 4 \\
$\underline{13}|24| 56$ & $4 \times 48$ & 6 & $\tau_{1}$ & 4 \\
$\underline{12}|36| 45$ & $4 \times 48$ & 6 & $\tau_{1}$ & 4 \\
$\underline{13}|25| 46$ & $8 \times 48$ & 7 & $\tau_{0}$ & 4 \\
$\underline{15}|26| 34$ & $8 \times 48$ & 7 & $\tau_{1}$ & 2 \\
$\underline{15}|23| 46$ & $16 \times 48$ & 8 & $\tau_{0}$ & 2 \\
\hline
\end{tabular}

We illustrate the procedure adopted to calculate the neighbours of a chamber in the chamber graph, selecting

$$
c_{10}:=\begin{array}{ll|ll|ll|}
6 & 4 & 3 & 2 & 5 & 4 \\
3 & 3 & 5 & 1 & 5 & 6 \\
5 & 1 & 2 & 2 & 1 & 6 \\
2 & 3 & 6 & 4 & 4 & 1 \\
\hline
\end{array}
$$

$\underline{12}|34| 56$

for demonstration purposes. Four of the ten neighbours of $c_{10}$ are readily obtained where the sextet is kept the same and the varying of the octad and trio is given by $12|\underline{34}| 56,12|34| \underline{56}, \underline{12}|35| 46$ and $\underline{12}|36| 45$. To locate the remaining six neighbours 
of $c_{10}$ (which will all have the same octad and trio as $c_{10}$ ), MOG comes out to play. Note that we are hunting for sextets whose tetrads are each contained in one of the octads of $T\left(c_{10}\right)$ as well as hitting two tetrads of $S\left(c_{10}\right)$ each in two elements. Let $t_{1}, \ldots, t_{6}$ denote the six tetrads of $S\left(c_{10}\right)$ and $O(1,2)=t_{1} \cup t_{2}, O(3,4)=$ $t_{3} \cup t_{4}, O(5,6)=t_{5} \cup t_{6}$, the three octads of $T\left(c_{10}\right)$.

First we select any 2 -element subset of $t_{1}$, say

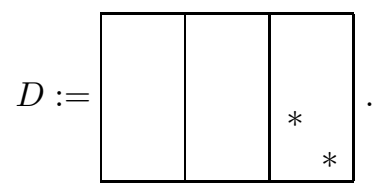

Now we look for an octad contained in $D \cup t_{2} \cup t_{3} \cup t_{4}$ and which contains $D$. Consulting the MOG gives

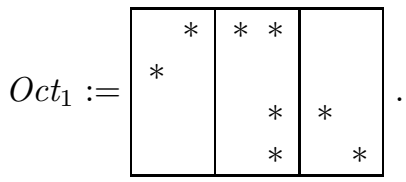

Then the intersection of $O c t_{1}$ with $O(1,2)$ and with $O(3,4)$ defines a partition of $O(1,2)$ and $O(3,4)$, and gives us four of the tetrads we seek, namely

$$
O c t_{1} \cap O(1,2), \quad O(1,2) \backslash O c t_{1}, \quad O c t_{1} \cap O(3,4), \quad O(3,4) \backslash O c t_{1} .
$$

We now repeat the above process, slightly modified, this time with the aim of finding an octad contained in $D \cup\left(t_{2} \cap O c t_{1}\right) \cup t_{5} \cup t_{6}$ which itself contains $D \cup\left(t_{2} \cap O c t_{1}\right)$. Using the MOG again we find

$$
\text { Oct }_{2}:=\begin{array}{|l|ll|ll|}
\hline & * & & * & \\
& & * & * & \\
& * & & & * \\
\hline
\end{array}
$$

Then $\mathrm{Oct}_{2} \cap O(5,6)$ and $O(5,6) \backslash \mathrm{Oct}_{2}$ supplies the remaining two tetrads we need to define the sextet

$$
\operatorname{Sex}_{1}:=\begin{array}{cc|cc|cc|}
\diamond & * & * & + & \circ & \times \\
* & \times & \circ & - & \diamond & \circ \\
\diamond & - & - & + & + & \diamond \\
- & \times & \circ & * & \times & + \\
\hline
\end{array} .
$$

REMARK. For each of $O c t_{1}$ and $O c t_{2}$ there is one other octad which also fulfils our requirements - however they will yield the same sextet. Also note that when finding $O c t_{1}$ we only needed to look in a certain 14-element subset of $\Omega$ and when finding $\mathrm{Oct}_{2}$ in a certain 12-element subset of $\Omega$. This, of course, makes our task easier.

Having pinpointed one sextet of the desired kind we enact the same procedure as above but starting with a 2 -element subset $D_{1}$ of a tetrad of $S\left(c_{10}\right)$ which is not contained in a tetrad of $S e x_{1}$. So we may select $D_{1}$ to be

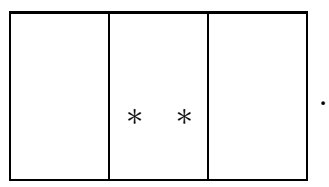


Then, with the aid of the MOG, we find that

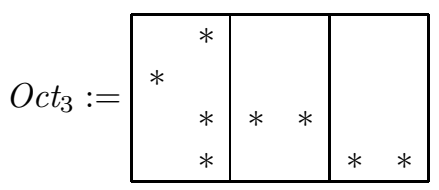

is an octad contained in $D_{1} \cup t_{1} \cup t_{3} \cup t_{4}$ which contains $D_{1}$. And then

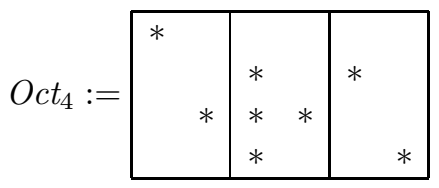

is an octad contained in $D_{1} \cup\left(t_{2} \cap O c t_{3}\right) \cup t_{5} \cup t_{6}$ which contains $D_{1} \cup\left(t_{2} \cap\right.$ Oct $\left._{3}\right)$. Using $\mathrm{Oct}_{3}$ and $\mathrm{Oct}_{4}$ we obtain the following sextet

$$
\text { Sex }_{2}:=\begin{array}{cc|cc|cc|}
\circ & \times & * & - & \diamond & * \\
\times & * & \circ & - & \circ & \diamond \\
\diamond & + & + & + & - & \diamond \\
- & \times & \circ & * & \times & + \\
\hline
\end{array} .
$$

A further sextet, $\mathrm{Sex}_{3}$, is obtained by taking the symmetric differences of the tetrads of $S e x_{1}$ and $S e x_{2}$.

$$
\operatorname{Sex}_{3}:=\begin{array}{cc|cc|cc|}
\circ & \times & \diamond & + & \circ & \times \\
\times & \times & * & - & \circ & 0 \\
* & + & + & - & + & * \\
- & \diamond & * & \diamond & \diamond & - \\
\hline
\end{array}
$$

By taking the symmetric differences of the tetrads of $S\left(c_{10}\right)$ and $S e x_{1}, S\left(c_{10}\right)$ and $S e x_{2}, S\left(c_{10}\right)$ and $S e x_{3}$ we produce the three remaining sextets of the required types. To summarize, the chambers which are neighbours of $c_{10}$ are

\begin{tabular}{|ll|ll|ll|}
\hline 6 & 4 & 3 & 3 & 5 & 4 \\
3 & 3 & 5 & 1 & 5 & 6 \\
5 & 1 & 2 & 2 & 1 & 6 \\
2 & 3 & 6 & 4 & 4 & 1 \\
\hline
\end{tabular}

$12|\underline{34}| 56$

\begin{tabular}{|ll|ll|ll|}
\hline 6 & 4 & 3 & 2 & 5 & 4 \\
3 & 3 & 5 & 1 & 5 & 6 \\
5 & 1 & 2 & 2 & 1 & 6 \\
2 & 3 & 6 & 4 & 4 & 1 \\
\hline
\end{tabular}

$12|34| \underline{56}$

\begin{tabular}{|ll|ll|ll|}
\hline 6 & 4 & 3 & 2 & 5 & 4 \\
3 & 3 & 5 & 1 & 5 & 6 \\
5 & 1 & 2 & 2 & 1 & 6 \\
2 & 3 & 6 & 4 & 4 & 1 \\
\hline
\end{tabular}

$\underline{12}|35| 46$

\begin{tabular}{|ll|ll|ll|}
\hline 6 & 4 & 3 & 2 & 5 & 4 \\
3 & 3 & 5 & 1 & 5 & 6 \\
5 & 1 & 2 & 2 & 1 & 6 \\
2 & 3 & 6 & 4 & 4 & 1 \\
\hline
\end{tabular}

$\underline{12}|36| 45$

\begin{tabular}{|ll|ll|ll|}
\hline 5 & 3 & 3 & 2 & 6 & 4 \\
3 & 4 & 6 & 1 & 5 & 6 \\
5 & 1 & 1 & 2 & 2 & 5 \\
1 & 4 & 6 & 3 & 4 & 2 \\
\hline
\end{tabular}

$\underline{12}|34| 56$

\begin{tabular}{|ll|ll|ll|}
\hline 5 & 3 & 4 & 1 & 6 & 4 \\
3 & 4 & 5 & 1 & 5 & 6 \\
6 & 2 & 2 & 2 & 1 & 6 \\
1 & 3 & 5 & 4 & 3 & 2 \\
\hline
\end{tabular}

$\underline{12}|34| 56$

\begin{tabular}{|ll|ll|ll|}
\hline 5 & 3 & 4 & 2 & 5 & 3 \\
3 & 3 & 6 & 1 & 5 & 5 \\
6 & 2 & 2 & 1 & 2 & 6 \\
1 & 4 & 6 & 4 & 4 & 1 \\
\hline
\end{tabular}

$\underline{12}|34| 56$

\begin{tabular}{|ll|ll|ll|}
\hline 5 & 4 & 3 & 2 & 5 & 3 \\
3 & 4 & 5 & 2 & 6 & 6 \\
6 & 2 & 1 & 2 & 1 & 5 \\
1 & 4 & 6 & 4 & 3 & 1 \\
\hline
\end{tabular}

$\underline{12}|34| 56$ 
and

\begin{tabular}{|ll|ll|ll|}
6 & 4 & 4 & 1 & 6 & 3 \\
3 & 4 & 5 & 2 & 5 & 5 \\
6 & 1 & 2 & 2 & 2 & 5 \\
1 & 3 & 6 & 3 & 4 & 1 \\
\hline
\end{tabular}

$\underline{12}|34| 56$

\begin{tabular}{|ll|ll|ll|}
5 & 4 & 4 & 2 & 6 & 4 \\
3 & 3 & 5 & 2 & 6 & 5 \\
5 & 1 & 2 & 1 & 1 & 6 \\
1 & 4 & 6 & 3 & 3 & 2 \\
\hline
\end{tabular}

$\underline{12}|34| 56$

Finally we note that by resorting the data in (3.2) we obtain the disc sizes stated in Theorem 1.

\section{Properties of $\mathcal{C}$}

Though (3.2) presents $\mathcal{C}$ from the viewpoint of $c_{0}$, it is an easy matter to translate this information to another chamber $c_{0}^{\prime}$. Then the first, third and last two columns would, respectively, give $G_{c_{0}^{\prime}}$-orbits, distance between $c_{0}^{\prime}$ and $c, \tau_{i}\left(T\left(c_{0}^{\prime}\right)\right)$ and $\left|O\left(c_{0}^{\prime}\right) \cap O(c)\right|(c$ a chamber of $\mathcal{C})$. So, for example, we may use (3.2) to discover the distance between two given chambers $c^{\prime}, c^{\prime \prime}$. Our elimination procedure being as follows:

(a) find $i$ such that $S\left(c^{\prime \prime}\right) \in \sigma_{i}\left(S\left(c^{\prime}\right)\right)$;

(b) using the $G_{c^{\prime}}$-orbits (obtained by suitably modifying (3.1)) find $n$ such that $S\left(c^{\prime \prime}\right) \in \sigma_{i}^{(n)}\left(S\left(c^{\prime}\right)\right)$

(c) narrow down the possibilities in the $\sigma_{i}^{(n)}$ table, using $T\left(c^{\prime \prime}\right) \in \tau_{k}\left(T\left(c^{\prime}\right)\right)$ and $\left|O\left(c^{\prime}\right) \cap O\left(c^{\prime \prime}\right)\right|$; and finally

(d) by examining the remaining options deduce to which $G_{c^{\prime}}$-orbit $c^{\prime \prime}$ belongs and thence obtain the distance between $c^{\prime}$ and $c^{\prime \prime}$.

We give a 'real life' illustration, taking our chambers to be

$$
c^{\prime}=\begin{array}{ll|ll|ll}
\hline 1 & 2 & 2 & 2 & 1 & 4 \\
6^{-} & 3^{+} & 4 & 5 & 5 & 1 \\
3 & 4^{+} & 6 & 5 & 3 & 2 \\
5^{-} & 3 & 6 & 4 & 6 & 1
\end{array} \quad, \quad c^{\prime \prime}=\begin{array}{|ll|ll|ll|}
5^{-} & 4^{+} & 3 & 4 & 4 & 2 \\
5 & 3^{+} & 6 & 6 & 3 & 2 \\
1 & 5 & 3 & 1 & 2 & 1 \\
6^{-} & 2 & 1 & 4 & 5 & 6 \\
\hline
\end{array}
$$

(a) $S\left(c^{\prime \prime}\right) \in \sigma_{0}\left(S\left(c^{\prime}\right)\right)$;

(b) (Here, of course, rather than mixed cols we should speak of mixed tetrads, where the tetrads are those of $S\left(c^{\prime}\right)$.) The mixed tetrads for $S\left(c^{\prime \prime}\right)$ are

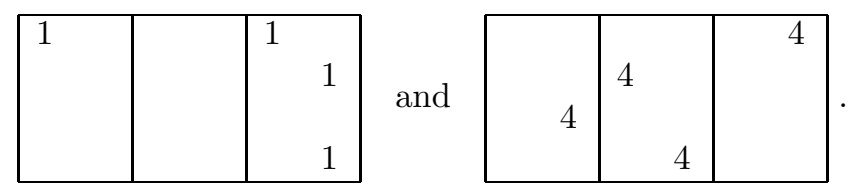

Since the first of these tetrads is in

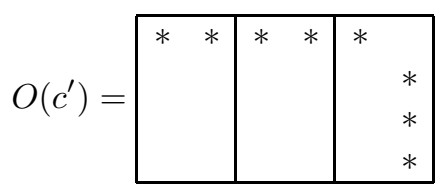

and the other is not, we see that $S\left(c^{\prime \prime}\right) \in \alpha_{0}^{(768)}\left(S\left(c^{\prime}\right)\right)$. 
(c) Since $T\left(c^{\prime \prime}\right) \in \tau_{0}\left(T\left(c^{\prime}\right)\right)$ and $\left|O\left(c^{\prime}\right) \cap O\left(c^{\prime \prime}\right)\right|=2$, one of the last eight possibilities of the $\sigma_{0}^{(768)}$ table in (3.2) must hold. Noting that $O\left(c^{\prime}\right) \cap O\left(c^{\prime \prime}\right)$ contains one element each from the two tetrads of $O\left(c^{\prime}\right)$ reduces the list of possibilities to four: $12|36| \underline{45}, 13|26| \underline{45}, \underline{12}|36| 45, \underline{12}|34| 56$. (Incidentally, at this stage we know that $c^{\prime}$ and $c^{\prime \prime}$ are either distance 9 or 10 apart.)

(d) We scrutinize the finer structure of the intersection of the octads of $T\left(c^{\prime \prime}\right)$ with $O\left(c^{\prime}\right)$. The octad of $T\left(c^{\prime \prime}\right)$,

\begin{tabular}{|l|ll|l|}
\hline$*$ & $*$ & $*$ & $*$ \\
$*$ & & & $*$ \\
& $*$ & & \\
& & $*$ & \\
\hline
\end{tabular}

intersects $O\left(c^{\prime}\right)$ in 4 elements which split $3 \mid 1$ between the tetrads of $O\left(c^{\prime}\right)$, and the octad of $T\left(c^{\prime \prime}\right)$,

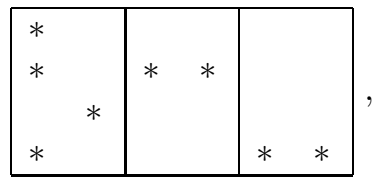

intersects $O\left(c^{\prime}\right)$ in two elements which are both contained in one of the tetrads of $O\left(c^{\prime}\right)$. Consequently, two possibilities remain, namely, $\underline{12}|34| 56$ and $13|26| \underline{45}$.

The octad of $T\left(c^{\prime}\right)$, not equal to $O\left(c^{\prime}\right)$, which intersects $O\left(c^{\prime \prime}\right)$ in two elements is

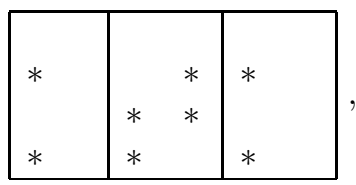

and the intersection this octad has with $O\left(c^{\prime \prime}\right)$ is contained in one of the tetrads of $O\left(c^{\prime \prime}\right)$. So this rules out $13|26| \underline{45}$ and therefore $\underline{12}|34| 56$ is the only possibility. Hence $d\left(c^{\prime}, c^{\prime \prime}\right)=10$.

In fact, $c^{\prime}$ and $c^{\prime \prime}$ are both distance 10 from $c_{0}$ as the reader may verify using the above scheme.

We recall, from Section 3, that

$c_{10}:=$\begin{tabular}{ll|ll|ll|}
6 & 4 & 3 & 2 & 5 & 4 \\
3 & 3 & 5 & 1 & 5 & 6 \\
5 & 1 & 2 & 2 & 1 & 6 \\
2 & 3 & 6 & 4 & 4 & 1 \\
\hline
\end{tabular}.

$\underline{12}|34| 56$

By (3.2) $c_{10} \in D_{10}\left(c_{0}\right),\left(c_{10}\right.$ is the representative of the $B$-orbit given in the $\sigma_{0}^{(768)}$ table). Before analysing the geodesic closure of $c_{0}$ and $c_{10}$, we note that (3.2) implies that $B=G_{c_{0}}$ is transitive on $D_{10}\left(c_{10}\right)$ and, combining (3.2) and (3.3), that $c_{10}$ has no neighbours in $D_{10}\left(c_{0}\right)$. This establishes Theorem 2. Turning to Theorem 3 , the following basic observation enables us, using (3.2), to enumerate the chambers 
in $\overline{\left\{c_{0}, c_{10}\right\}}$ : for $j$ with $0 \leqslant j \leqslant 10$,

$$
\overline{\left\{c_{0}, c_{10}\right\}} \cap D_{j}\left(c_{0}\right)=\overline{\left\{c_{0}, c_{10}\right\}} \cap D_{10-j}\left(c_{10}\right)=D_{j}\left(c_{0}\right) \cap D_{10-j}\left(c_{10}\right) .
$$

Set $\Delta_{j}=\overline{\left\{c_{0}, c_{10}\right\}} \cap D_{j}\left(c_{0}\right)$. Put

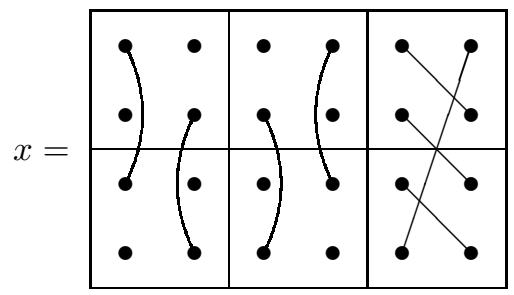

and

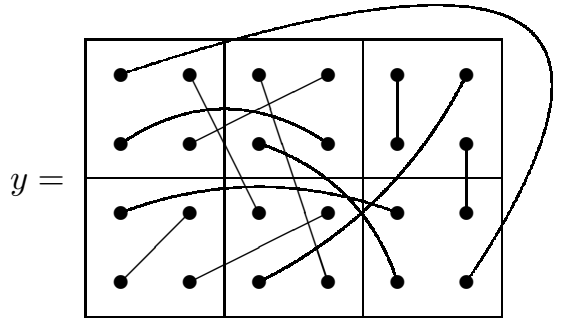

Then $x, y \in G$ (this may be checked using [5]) and $x^{2}=y^{2}=1$. (We point out that $x$ may be described by the octad it fixes point-wise and any one of the pairs of points it interchanges. Whereas $y$ is an example of an involution given by the recipe: leave invariant the tetrads of a sextet and a dodecad which cuts across the sextet in $2^{6}$.) Moreover, $x$ fixes $c_{0}$ and $c_{10}$ (so $\left.B_{c_{10}}=\langle x\rangle\right)$ and $y$ interchanges $c_{0}$ and $c_{10}$. Thus $y$ interchanges the sets $\Delta_{j}$ and $\Delta_{10-j}$ for $0 \leqslant j \leqslant 10$. In Appendix A we list the chambers in $\overline{\left\{c_{0}, c_{10}\right\}}$. There we label each chamber by $c_{j}^{k}-j$ indicates that $c_{j}^{k}$ is in the $j$ th disc of $c_{0}$ (that is $c_{j}^{k} \in D_{j}\left(c_{0}\right)$ ) and the $k$ is just a superscript. Our notation is arranged so as, when $j \neq 5, y$ maps $c_{k}^{j}$ to $c_{k}^{10-j}$. The existence of $y$ means that it is only necessary for us to draw just over 'half' of $\overline{\left\{c_{0}, c_{10}\right\}}$; this we do in Appendix B.

To complete the story of the action of $y$ upon $\overline{\left\{c_{0}, c_{10}\right\}}$ we have

(4.1) The orbits of $y$ upon $\Delta_{5}$ are $\left\{c_{5}^{1}, c_{5}^{14}\right\},\left\{c_{5}^{2}, c_{5}^{3}\right\},\left\{c_{5}^{4}, c_{5}^{5}\right\},\left\{c_{5}^{6}, c_{5}^{9}\right\},\left\{c_{5}^{7}, c_{5}^{8}\right\}$, $\left\{c_{5}^{10}, c_{5}^{13}\right\},\left\{c_{5}^{11}, c_{5}^{12}\right\}$.

REMARK. $y$ fixes no chambers in $\overline{\left\{c_{0}, c_{10}\right\}}$.

We also document the action of $x$ upon $\overline{\left\{c_{0}, c_{10}\right\}}$. Since $x$ and $y$ commute, we only need consider $\Delta_{i}$ for $1 \leqslant i \leqslant 5$.

(4.2) The orbits of $x$ upon $\Delta_{i}$ are as follows:

$$
\begin{aligned}
& i=1:\left\{c_{1}^{1}\right\},\left\{c_{1}^{6}\right\},\left\{c_{1}^{9}\right\},\left\{c_{1}^{10}\right\},\left\{c_{1}^{2}, c_{1}^{4}\right\},\left\{c_{1}^{3}, c_{1}^{5}\right\},\left\{c_{1}^{7}, c_{1}^{8}\right\} \\
& i=2:\left\{c_{2}^{1}\right\},\left\{c_{2}^{6}\right\},\left\{c_{2}^{7}\right\},\left\{c_{2}^{12}\right\},\left\{c_{2}^{2}, c_{2}^{4}\right\},\left\{c_{2}^{3}, c_{2}^{5}\right\},\left\{c_{2}^{8}, c_{2}^{10}\right\},\left\{c_{2}^{9}, c_{2}^{11}\right\} \\
& i=3:\left\{c_{3}^{1}\right\},\left\{c_{3}^{8}\right\},\left\{c_{3}^{9}\right\},\left\{c_{3}^{10}\right\},\left\{c_{3}^{13}\right\},\left\{c_{3}^{14}\right\},\left\{c_{3}^{2}, c_{3}^{5}\right\},\left\{c_{3}^{3}, c_{3}^{6}\right\},\left\{c_{3}^{4}, c_{3}^{7}\right\},\left\{c_{3}^{11}, c_{3}^{12}\right\} \\
& i=4: \quad\left\{c_{4}^{1}\right\},\left\{c_{4}^{2}\right\},\left\{c_{4}^{9}\right\},\left\{c_{4}^{10}\right\},\left\{c_{4}^{11}\right\},\left\{c_{4}^{12}\right\},\left\{c_{4}^{15}\right\},\left\{c_{4}^{3}, c_{4}^{6}\right\},\left\{c_{4}^{4}, c_{4}^{7}\right\},\left\{c_{4}^{5}, c_{4}^{8}\right\}, \\
&\left\{c_{4}^{13}, c_{4}^{14}\right\} \\
& i=5:\left\{c_{5}^{1}\right\},\left\{c_{5}^{6}\right\},\left\{c_{5}^{7}\right\},\left\{c_{5}^{8}\right\},\left\{c_{5}^{9}\right\},\left\{c_{5}^{14}\right\},\left\{c_{5}^{2}, c_{5}^{4}\right\},\left\{c_{5}^{3}, c_{5}^{5}\right\},\left\{c_{5}^{10}, c_{5}^{12}\right\},\left\{c_{5}^{11}, c_{5}^{13}\right\} .
\end{aligned}
$$




\section{Appendix A. The chambers in $\overline{\left\{c_{0}, c_{10}\right\}}$}

Each of the sextets listed below are chambers via $\underline{12}|34| 56$.

$\Delta_{0}:$

$$
c_{0}=\begin{array}{ll|ll|ll|}
1 & 2 & 3 & 4 & 5 & 6 \\
1 & 2 & 3 & 4 & 5 & 6 \\
1 & 2 & 3 & 4 & 5 & 6 \\
1 & 2 & 3 & 4 & 5 & 6 \\
\hline
\end{array}
$$

$\Delta_{1}:$

$$
\begin{aligned}
& c_{1}^{1}=\begin{array}{ll|ll|ll}
1 & 1 & 4 & 4 & 6 & 6 \\
2 & 2 & 3 & 3 & 5 & 5 \\
1 & 1 & 4 & 4 & 6 & 6 \\
2 & 2 & 3 & 3 & 5 & 5 \\
\hline
\end{array} \\
& c_{1}^{2}=\begin{array}{ll|ll|ll}
1 & 1 & 3 & 3 & 6 & 6 \\
1 & 1 & 3 & 3 & 6 & 6 \\
2 & 2 & 4 & 4 & 5 & 5 \\
2 & 2 & 4 & 4 & 5 & 5 \\
\hline
\end{array} \\
& c_{1}^{4}=\begin{array}{ll|ll|ll}
1 & 2 & 3 & 4 & 5 & 6 \\
2 & 1 & 4 & 3 & 6 & 5 \\
2 & 1 & 4 & 3 & 6 & 5 \\
1 & 2 & 3 & 4 & 5 & 6 \\
\hline
\end{array} \\
& c_{1}^{7}=\begin{array}{ll|ll|ll}
1 & 2 & 6 & 3 & 4 & 5 \\
1 & 2 & 6 & 3 & 4 & 5 \\
1 & 2 & 6 & 3 & 4 & 5 \\
1 & 2 & 6 & 3 & 4 & 5 \\
\hline
\end{array} \\
& c_{1}^{10}=\begin{array}{ll|ll|ll|}
6 & 5 & 4 & 3 & 1 & 2 \\
6 & 5 & 4 & 3 & 1 & 2 \\
6 & 5 & 4 & 3 & 1 & 2 \\
6 & 5 & 4 & 3 & 1 & 2 \\
\hline
\end{array}
\end{aligned}
$$

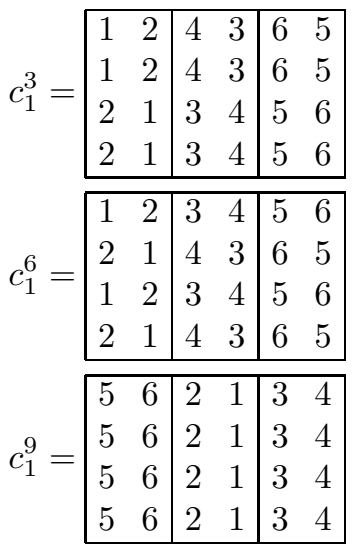

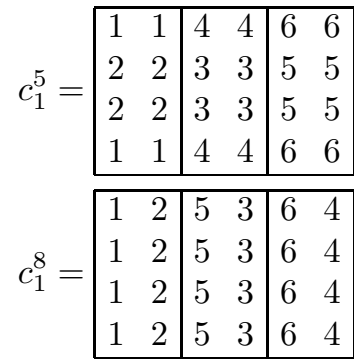

$\Delta_{2}:$

$$
\begin{aligned}
& c_{2}^{1}=\begin{array}{ll|ll|ll}
5 & 5 & 4 & 4 & 1 & 1 \\
6 & 6 & 3 & 3 & 2 & 2 \\
5 & 5 & 4 & 4 & 1 & 1 \\
6 & 6 & 3 & 3 & 2 & 2 \\
\hline
\end{array} \\
& c_{2}^{4}=\begin{array}{ll|ll|ll}
6 & 5 & 1 & 2 & 3 & 4 \\
5 & 6 & 2 & 1 & 4 & 3 \\
5 & 6 & 2 & 1 & 4 & 3 \\
6 & 5 & 1 & 2 & 3 & 4 \\
\hline
\end{array} \\
& c_{2}^{7}=\begin{array}{ll|ll|ll|}
5 & 6 & 1 & 2 & 3 & 4 \\
6 & 5 & 2 & 1 & 4 & 3 \\
5 & 6 & 1 & 2 & 3 & 4 \\
6 & 5 & 2 & 1 & 4 & 3 \\
\hline
\end{array} \\
& c_{2}^{10}=\begin{array}{ll|ll|ll}
1 & 1 & 5 & 3 & 5 & 3 \\
2 & 2 & 5 & 3 & 5 & 3 \\
2 & 1 & 6 & 4 & 6 & 4 \\
1 & 2 & 6 & 4 & 6 & 4 \\
\hline
\end{array}
\end{aligned}
$$
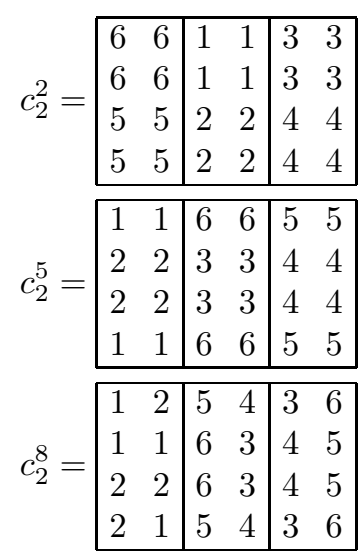

$$
c_{2}^{11}=\begin{array}{ll|ll|ll|}
6 & 5 & 4 & 1 & 3 & 2 \\
6 & 5 & 4 & 1 & 3 & 2 \\
6 & 5 & 4 & 1 & 3 & 2 \\
6 & 5 & 4 & 1 & 3 & 2 \\
\hline
\end{array}
$$

$$
\begin{aligned}
& c_{2}^{3}=\begin{array}{ll|ll|ll|}
1 & 2 & 5 & 3 & 4 & 6 \\
1 & 2 & 5 & 3 & 4 & 6 \\
2 & 1 & 3 & 5 & 6 & 4 \\
2 & 1 & 3 & 5 & 6 & 4 \\
\hline
\end{array} \\
& c_{2}^{6}=\begin{array}{ll|ll|ll|}
1 & 2 & 3 & 5 & 6 & 4 \\
2 & 1 & 5 & 3 & 4 & 6 \\
1 & 2 & 3 & 5 & 6 & 4 \\
2 & 1 & 5 & 3 & 4 & 6 \\
\hline
\end{array} \\
& c_{2}^{9}=\begin{array}{ll|ll|ll|}
6 & 5 & 3 & 1 & 2 & 4 \\
6 & 5 & 3 & 1 & 2 & 4 \\
6 & 5 & 3 & 1 & 2 & 4 \\
6 & 5 & 3 & 1 & 2 & 4 \\
\hline
\end{array} \\
& c_{2}^{12}=\begin{array}{ll|ll|ll|}
\hline 5 & 4 & 6 & 3 & 1 & 2 \\
5 & 4 & 6 & 3 & 1 & 2 \\
5 & 4 & 6 & 3 & 1 & 2 \\
5 & 4 & 6 & 3 & 1 & 2 \\
\hline
\end{array}
\end{aligned}
$$


$\Delta_{3}:$

$$
\begin{aligned}
& c_{3}^{1}=\begin{array}{|ll|ll|ll}
4 & 4 & 6 & 6 & 1 & 1 \\
5 & 5 & 3 & 3 & 2 & 2 \\
4 & 4 & 6 & 6 & 1 & 1 \\
5 & 5 & 3 & 3 & 2 & 2 \\
\hline
\end{array} \\
& c_{3}^{2}=\begin{array}{ll|ll|ll|}
4 & 4 & 1 & 1 & 3 & 3 \\
4 & 4 & 1 & 1 & 3 & 3 \\
6 & 6 & 2 & 2 & 5 & 5 \\
6 & 6 & 2 & 2 & 5 & 5 \\
\hline
\end{array} \\
& c_{3}^{4}=\begin{array}{ll|ll|ll}
6 & 5 & 3 & 1 & 2 & 4 \\
6 & 5 & 3 & 1 & 2 & 4 \\
5 & 6 & 1 & 3 & 4 & 2 \\
5 & 6 & 1 & 3 & 4 & 2 \\
\hline
\end{array} \\
& c_{3}^{7}=\begin{array}{lll|ll|ll|}
5 & 5 & 4 & 4 & 3 & 3 \\
6 & 6 & 1 & 1 & 2 & 2 \\
6 & 6 & 1 & 1 & 2 & 2 \\
5 & 5 & 4 & 4 & 3 & 3 \\
\hline
\end{array} \\
& c_{3}^{10}=\begin{array}{|ll|ll|ll|}
6 & 4 & 1 & 2 & 3 & 5 \\
4 & 6 & 2 & 1 & 5 & 3 \\
6 & 4 & 1 & 2 & 3 & 5 \\
4 & 6 & 2 & 1 & 5 & 3 \\
\hline
\end{array} \\
& c_{3}^{13}=\begin{array}{|ll|ll|ll|}
6 & 3 & 5 & 4 & 1 & 2 \\
5 & 4 & 6 & 3 & 1 & 1 \\
6 & 3 & 5 & 4 & 2 & 1 \\
5 & 4 & 6 & 3 & 2 & 2 \\
\hline
\end{array} \\
& c_{3}^{5}=\begin{array}{|ll|ll|ll|}
\hline 5 & 4 & 1 & 2 & 3 & 6 \\
4 & 5 & 2 & 1 & 6 & 3 \\
4 & 5 & 2 & 1 & 6 & 3 \\
5 & 4 & 1 & 2 & 3 & 6 \\
\hline
\end{array} \\
& c_{3}^{8}=\begin{array}{ll|ll|ll|}
1 & 2 & 4 & 6 & 6 & 4 \\
2 & 2 & 5 & 3 & 3 & 5 \\
1 & 1 & 3 & 5 & 5 & 3 \\
1 & 2 & 6 & 4 & 4 & 6 \\
\hline
\end{array} \\
& c_{3}^{11}=\begin{array}{ll|ll|ll|}
5 & 6 & 3 & 2 & 1 & 4 \\
5 & 5 & 4 & 1 & 2 & 3 \\
6 & 6 & 4 & 1 & 2 & 3 \\
6 & 5 & 3 & 2 & 1 & 4 \\
\hline
\end{array} \\
& c_{3}^{14}=\begin{array}{lll|ll|ll|}
6 & 2 & 5 & 1 & 3 & 4 \\
6 & 2 & 5 & 1 & 3 & 4 \\
6 & 2 & 5 & 1 & 3 & 4 \\
6 & 2 & 5 & 1 & 3 & 4 \\
\hline
\end{array} \\
& c_{3}^{3}=\begin{array}{ll|ll|ll|}
1 & 2 & 5 & 4 & 4 & 5 \\
2 & 2 & 6 & 3 & 3 & 6 \\
1 & 1 & 3 & 6 & 6 & 3 \\
1 & 2 & 4 & 5 & 5 & 4 \\
\hline
\end{array} \\
& c_{3}^{6}=\begin{array}{|ll|ll|ll|}
1 & 2 & 6 & 5 & 5 & 6 \\
2 & 2 & 4 & 3 & 3 & 4 \\
1 & 1 & 3 & 4 & 4 & 3 \\
1 & 2 & 5 & 6 & 6 & 5 \\
\hline
\end{array} \\
& c_{3}^{9}=\begin{array}{|ll|ll|ll|}
\hline 5 & 6 & 3 & 2 & 1 & 4 \\
6 & 5 & 2 & 3 & 4 & 1 \\
5 & 6 & 3 & 2 & 1 & 4 \\
6 & 5 & 2 & 3 & 4 & 1 \\
\hline
\end{array} \\
& c_{3}^{12}=\begin{array}{|ll|ll|ll|}
\hline 6 & 6 & 3 & 1 & 3 & 1 \\
5 & 5 & 3 & 1 & 3 & 1 \\
5 & 6 & 4 & 2 & 4 & 2 \\
6 & 5 & 4 & 2 & 4 & 2 \\
\hline
\end{array}
\end{aligned}
$$

$\Delta_{4}:$

$$
\begin{aligned}
& c_{4}^{1}=\begin{array}{ll|ll|ll|}
4 & 3 & 6 & 5 & 1 & 2 \\
6 & 5 & 4 & 3 & 1 & 1 \\
4 & 3 & 6 & 5 & 2 & 1 \\
6 & 5 & 4 & 3 & 2 & 2 \\
\hline
\end{array} \\
& c_{4}^{4}=\begin{array}{|ll|ll|ll|}
2 & 2 & 3 & 3 & 1 & 1 \\
2 & 2 & 3 & 3 & 1 & 1 \\
5 & 5 & 4 & 4 & 6 & 6 \\
5 & 5 & 4 & 4 & 6 & 6 \\
\hline
\end{array} \\
& c_{4}^{7}=\begin{array}{lll|ll|ll|}
6 & 2 & 3 & 4 & 1 & 5 \\
2 & 6 & 4 & 3 & 5 & 1 \\
2 & 6 & 4 & 3 & 5 & 1 \\
6 & 2 & 3 & 4 & 1 & 5 \\
\hline
\end{array} \\
& c_{4}^{10}=\begin{array}{lll|ll|ll|}
6 & 4 & 3 & 2 & 1 & 5 \\
4 & 6 & 2 & 3 & 5 & 1 \\
6 & 4 & 3 & 2 & 1 & 5 \\
4 & 6 & 2 & 3 & 5 & 1 \\
\hline
\end{array} \\
& c_{4}^{13}=\begin{array}{lll|ll|ll|}
4 & 5 & 3 & 2 & 1 & 6 \\
4 & 4 & 6 & 1 & 2 & 3 \\
5 & 5 & 6 & 1 & 2 & 3 \\
5 & 4 & 3 & 2 & 1 & 6 \\
\hline
\end{array}
\end{aligned}
$$

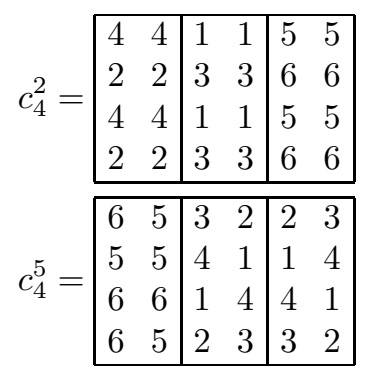

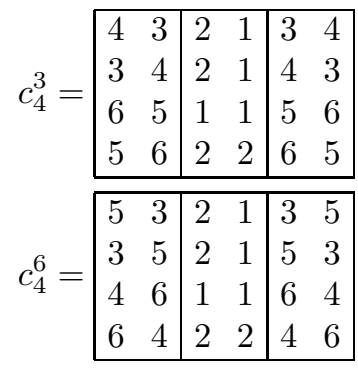$$
c_{4}^{8}=\begin{array}{|ll|ll|ll|}
6 & 5 & 4 & 3 & 3 & 4 \\
5 & 5 & 2 & 1 & 1 & 2 \\
6 & 6 & 1 & 2 & 2 & 1 \\
6 & 5 & 3 & 4 & 4 & 3 \\
\hline
\end{array}
$$$$
c_{4}^{9}=\begin{array}{|ll|ll|ll|}
6 & 5 & 4 & 1 & 1 & 4 \\
5 & 5 & 2 & 3 & 3 & 2 \\
6 & 6 & 3 & 2 & 2 & 3 \\
6 & 5 & 1 & 4 & 4 & 1 \\
\hline
\end{array}
$$$$
c_{4}^{11}=\begin{array}{|ll|ll|ll|}
6 & 3 & 2 & 1 & 3 & 6 \\
3 & 6 & 2 & 1 & 6 & 3 \\
5 & 4 & 1 & 1 & 4 & 5 \\
4 & 5 & 2 & 2 & 5 & 4 \\
\hline
\end{array}
$$$$
c_{4}^{14}=\begin{array}{ll|ll|ll|}
5 & 5 & 3 & 1 & 3 & 1 \\
4 & 4 & 3 & 1 & 3 & 1 \\
4 & 5 & 6 & 2 & 6 & 2 \\
5 & 4 & 6 & 2 & 6 & 2 \\
\hline
\end{array}
$$

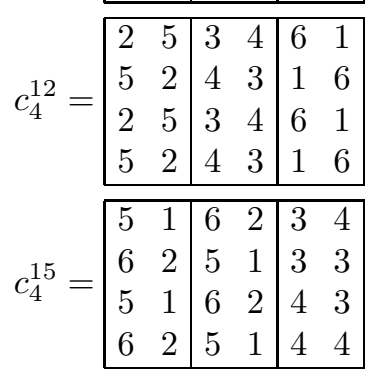


$\Delta_{5}$ :

$$
\begin{aligned}
& c_{5}^{1}=\begin{array}{ll|ll|ll}
5 & 1 & 3 & 2 & 6 & 4 \\
3 & 2 & 5 & 1 & 6 & 6 \\
5 & 1 & 3 & 2 & 4 & 6 \\
3 & 2 & 5 & 1 & 4 & 4
\end{array} \quad c_{5}^{2}=\begin{array}{lll|ll|ll}
6 & 3 & 4 & 2 & 2 & 4 \\
3 & 3 & 5 & 1 & 1 & 5 \\
6 & 6 & 1 & 5 & 5 & 1 \\
6 & 3 & 2 & 4 & 4 & 2 \\
\hline
\end{array} \quad c_{5}^{3}=\begin{array}{lll|ll|ll|}
2 & 1 & 3 & 4 & 1 & 2 \\
1 & 2 & 3 & 4 & 2 & 1 \\
6 & 5 & 4 & 4 & 5 & 6 \\
5 & 6 & 3 & 3 & 6 & 5 \\
\hline
\end{array} \\
& c_{5}^{4}=\begin{array}{ll|ll|ll}
6 & 3 & 4 & 5 & 5 & 4 \\
3 & 3 & 2 & 1 & 1 & 2 \\
6 & 6 & 1 & 2 & 2 & 1 \\
6 & 3 & 5 & 4 & 4 & 5
\end{array} \quad \quad c_{5}^{5}=\begin{array}{lll|ll|ll}
3 & 1 & 5 & 6 & 1 & 3 \\
1 & 3 & 5 & 6 & 3 & 1 \\
2 & 4 & 6 & 6 & 4 & 2 \\
4 & 2 & 5 & 5 & 2 & 4 \\
\hline
\end{array} \quad c_{5}^{6}=\begin{array}{lll|ll|ll|}
1 & 3 & 5 & 6 & 3 & 1 \\
3 & 1 & 5 & 6 & 1 & 3 \\
2 & 4 & 6 & 6 & 4 & 2 \\
4 & 2 & 5 & 5 & 2 & 4 \\
\hline
\end{array} \\
& c_{5}^{7}=\begin{array}{|ll|ll|ll}
2 & 3 & 4 & 6 & 5 & 1 \\
3 & 2 & 6 & 4 & 1 & 5 \\
2 & 3 & 4 & 6 & 5 & 1 \\
3 & 2 & 6 & 4 & 1 & 5
\end{array} \quad c_{5}^{8}=\begin{array}{lll|ll|ll|}
5 & 3 & 3 & 2 & 1 & 6 \\
3 & 6 & 1 & 4 & 5 & 1 \\
5 & 4 & 4 & 2 & 2 & 5 \\
4 & 6 & 1 & 3 & 6 & 2 \\
\hline
\end{array} \\
& c_{5}^{10}=\begin{array}{ll|ll|ll}
6 & 6 & 2 & 4 & 2 & 4 \\
1 & 1 & 2 & 4 & 2 & 4 \\
1 & 6 & 5 & 3 & 5 & 3 \\
6 & 1 & 5 & 3 & 5 & 3
\end{array} \quad \quad c_{5}^{11}=\begin{array}{lll|ll|ll}
6 & 6 & 4 & 2 & 4 & 1 \\
3 & 4 & 3 & 2 & 3 & 1 \\
4 & 5 & 5 & 2 & 5 & 1 \\
5 & 3 & 6 & 1 & 6 & 2 \\
\hline
\end{array} \quad c_{5}^{12}=\begin{array}{lllll|ll|}
1 & 5 & 2 & 3 & 4 & 6 \\
1 & 1 & 6 & 4 & 3 & 2 \\
5 & 5 & 6 & 4 & 3 & 2 \\
5 & 1 & 2 & 3 & 4 & 6 \\
\hline
\end{array} \\
& c_{5}^{13}=\begin{array}{ll|ll|ll}
4 & 5 & 4 & 2 & 1 & 5 \\
3 & 3 & 5 & 2 & 1 & 4 \\
5 & 6 & 6 & 2 & 2 & 3 \\
6 & 4 & 3 & 1 & 1 & 6
\end{array} \quad c_{5}^{14}=\begin{array}{lll|ll|ll|}
6 & 5 & 1 & 2 & 3 & 4 \\
1 & 2 & 6 & 5 & 3 & 3 \\
6 & 5 & 1 & 2 & 4 & 3 \\
1 & 2 & 6 & 5 & 4 & 4 \\
\hline
\end{array}
\end{aligned}
$$

$\Delta_{6}$ :

$$
\begin{aligned}
& c_{6}^{1}=\begin{array}{ll|ll|ll}
2 & 6 & 3 & 5 & 1 & 4 \\
3 & 5 & 2 & 6 & 1 & 1 \\
2 & 6 & 3 & 5 & 4 & 1 \\
3 & 5 & 2 & 6 & 4 & 4 \\
\hline
\end{array} \\
& c_{6}^{4}=\begin{array}{ll|ll|ll|}
6 & 4 & 4 & 2 & 1 & 4 \\
3 & 3 & 6 & 2 & 1 & 6 \\
6 & 5 & 2 & 5 & 5 & 1 \\
5 & 4 & 1 & 3 & 3 & 2 \\
\hline
\end{array} \\
& c_{6}^{7}=\begin{array}{ll|ll|ll}
5 & 4 & 4 & 6 & 5 & 3 \\
3 & 4 & 1 & 2 & 1 & 1 \\
5 & 6 & 2 & 2 & 2 & 1 \\
6 & 3 & 5 & 3 & 4 & 6 \\
\hline
\end{array} \\
& c_{6}^{10}=\begin{array}{ll|ll|ll|}
1 & 3 & 3 & 6 & 5 & 2 \\
3 & 2 & 5 & 4 & 1 & 5 \\
1 & 4 & 4 & 6 & 6 & 1 \\
4 & 2 & 5 & 3 & 2 & 6 \\
\hline
\end{array} \\
& c_{6}^{13}=\begin{array}{ll|ll|ll}
6 & 6 & 2 & 4 & 2 & 3 \\
1 & 2 & 1 & 4 & 1 & 3 \\
2 & 5 & 5 & 4 & 5 & 3 \\
5 & 1 & 6 & 3 & 6 & 4 \\
\hline
\end{array}
\end{aligned}
$$

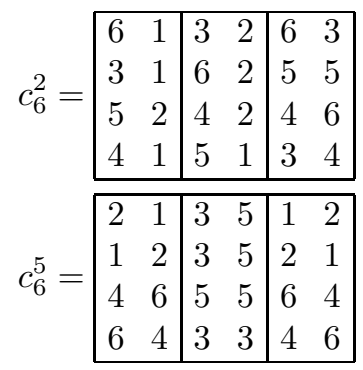

$$
\begin{aligned}
& c_{6}^{3}=\begin{array}{ll|ll|ll|}
5 & 1 & 2 & 4 & 4 & 2 \\
1 & 1 & 6 & 3 & 3 & 6 \\
5 & 5 & 3 & 6 & 6 & 3 \\
5 & 1 & 4 & 2 & 2 & 4 \\
\hline
\end{array} \\
& c_{6}^{6}=\begin{array}{ll|ll|ll|}
6 & 1 & 2 & 5 & 5 & 2 \\
1 & 1 & 4 & 3 & 3 & 4 \\
6 & 6 & 3 & 4 & 4 & 3 \\
6 & 1 & 5 & 2 & 2 & 5 \\
\hline
\end{array} \\
& c_{6}^{8}=\begin{array}{ll|ll|ll|}
3 & 1 & 4 & 5 & 1 & 3 \\
1 & 3 & 4 & 5 & 3 & 1 \\
2 & 6 & 5 & 5 & 6 & 2 \\
6 & 2 & 4 & 4 & 2 & 6 \\
\hline
\end{array} \\
& c_{6}^{11}=\begin{array}{ll|ll|ll}
4 & 1 & 2 & 6 & 6 & 2 \\
1 & 1 & 5 & 3 & 3 & 5 \\
4 & 4 & 3 & 5 & 5 & 3 \\
4 & 1 & 6 & 2 & 2 & 6 \\
\hline
\end{array} \\
& c_{6}^{14}=\begin{array}{ll|ll|ll}
2 & 6 & 2 & 4 & 3 & 6 \\
1 & 1 & 6 & 4 & 3 & 2 \\
6 & 5 & 5 & 4 & 4 & 1 \\
5 & 2 & 1 & 3 & 3 & 5 \\
\hline
\end{array} \\
& c_{6}^{9}=\begin{array}{ll|ll|ll|}
1 & 3 & 4 & 5 & 3 & 1 \\
3 & 1 & 4 & 5 & 1 & 3 \\
2 & 6 & 5 & 5 & 6 & 2 \\
6 & 2 & 4 & 4 & 2 & 6 \\
\hline
\end{array} \\
& c_{6}^{12}=\begin{array}{|ll|ll|ll|}
6 & 3 & 3 & 2 & 1 & 4 \\
3 & 4 & 1 & 5 & 6 & 1 \\
6 & 5 & 5 & 2 & 2 & 6 \\
5 & 4 & 1 & 3 & 4 & 2 \\
\hline
\end{array} \\
& c_{6}^{15}=\begin{array}{ll|ll|ll}
4 & 6 & 1 & 2 & 3 & 5 \\
1 & 2 & 4 & 6 & 3 & 3 \\
4 & 6 & 1 & 2 & 5 & 3 \\
1 & 2 & 4 & 6 & 5 & 5 \\
\hline
\end{array}
\end{aligned}
$$


$\Delta_{7}:$

$$
\begin{aligned}
& c_{7}^{1}=\begin{array}{ll|ll|ll}
2 & 6 & 3 & 5 & 2 & 3 \\
3 & 6 & 2 & 5 & 1 & 1 \\
1 & 5 & 4 & 5 & 4 & 2 \\
4 & 6 & 1 & 6 & 3 & 4 \\
\hline
\end{array} \\
& c_{7}^{4}=\begin{array}{ll|ll|ll}
2 & 1 & 3 & 5 & 2 & 1 \\
1 & 1 & 4 & 6 & 2 & 2 \\
4 & 5 & 5 & 6 & 5 & 4 \\
6 & 3 & 4 & 3 & 3 & 6 \\
\hline
\end{array} \quad c_{7}^{5}=\begin{array}{lll|ll|ll|}
6 & 2 & 2 & 5 & 6 & 1 \\
1 & 2 & 3 & 4 & 3 & 3 \\
6 & 5 & 4 & 4 & 4 & 3 \\
5 & 1 & 6 & 1 & 2 & 5 \\
\hline
\end{array} \\
& c_{7}^{7}=\begin{array}{|ll|ll|ll}
3 & 1 & 4 & 6 & 2 & 4 \\
1 & 4 & 3 & 6 & 3 & 2 \\
2 & 5 & 5 & 5 & 6 & 2 \\
6 & 1 & 3 & 4 & 1 & 5 \\
\hline
\end{array} \quad \quad c_{7}^{8}=\begin{array}{|ll|ll|ll|}
6 & 3 & 4 & 2 & 3 & 6 \\
3 & 6 & 4 & 2 & 6 & 3 \\
5 & 1 & 2 & 2 & 1 & 5 \\
1 & 5 & 4 & 4 & 5 & 1 \\
\hline
\end{array} \\
& c_{7}^{10}=\begin{array}{|ll|ll|ll|}
3 & 1 & 1 & 6 & 5 & 2 \\
1 & 2 & 5 & 4 & 3 & 5 \\
3 & 4 & 4 & 6 & 6 & 3 \\
4 & 2 & 5 & 1 & 2 & 6 \\
\hline
\end{array} \\
& c_{7}^{13}=\begin{array}{lll|ll|ll}
2 & 5 & 3 & 4 & 1 & 6 \\
3 & 4 & 2 & 5 & 1 & 1 \\
2 & 5 & 3 & 4 & 6 & 1 \\
3 & 4 & 2 & 5 & 6 & 6
\end{array} \quad \quad c_{7}^{14}=\begin{array}{lll|ll|ll|}
4 & 5 & 1 & 2 & 3 & 5 \\
1 & 1 & 3 & 6 & 3 & 4 \\
3 & 6 & 2 & 2 & 6 & 4 \\
2 & 1 & 4 & 5 & 5 & 6 \\
\hline
\end{array}
\end{aligned}
$$

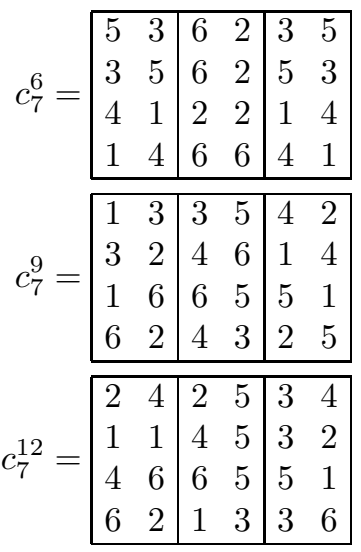

$$
\begin{aligned}
& c_{7}^{11}=\begin{array}{|ll|ll|ll}
4 & 4 & 2 & 5 & 2 & 3 \\
1 & 2 & 1 & 5 & 1 & 3 \\
2 & 6 & 6 & 5 & 6 & 3 \\
6 & 1 & 4 & 3 & 4 & 5 \\
\hline 4
\end{array} \quad c_{7}^{12}=\begin{array}{lllll|ll|}
2 & 4 & 2 & 5 & 3 & 4 \\
1 & 1 & 4 & 5 & 3 & 2 \\
4 & 6 & 6 & 5 & 5 & 1 \\
6 & 2 & 1 & 3 & 3 & 6 \\
\hline
\end{array}
\end{aligned}
$$

$\Delta_{8}:$

$$
\begin{aligned}
& c_{8}^{1}=\begin{array}{ll|ll|ll}
2 & 4 & 3 & 6 & 2 & 3 \\
3 & 4 & 2 & 6 & 1 & 1 \\
1 & 6 & 5 & 6 & 5 & 2 \\
5 & 4 & 1 & 4 & 3 & 5 \\
\hline
\end{array} \\
& c_{8}^{4}=\begin{array}{lll|ll|ll|}
4 & 2 & 2 & 6 & 4 & 1 \\
1 & 2 & 3 & 5 & 3 & 3 \\
4 & 6 & 5 & 5 & 5 & 3 \\
6 & 1 & 4 & 1 & 2 & 6 \\
\hline
\end{array} \\
& c_{8}^{7}=\begin{array}{ll|ll|ll|}
3 & 1 & 1 & 5 & 4 & 2 \\
1 & 2 & 4 & 6 & 3 & 4 \\
3 & 6 & 6 & 5 & 5 & 3 \\
6 & 2 & 4 & 1 & 2 & 5 \\
\hline
\end{array} \\
& c_{8}^{10}=\begin{array}{lll|ll|ll|}
4 & 6 & 4 & 2 & 5 & 6 \\
3 & 3 & 6 & 2 & 5 & 4 \\
6 & 1 & 1 & 2 & 2 & 3 \\
1 & 4 & 3 & 5 & 5 & 1 \\
\hline
\end{array} \\
& c_{8}^{2}=\begin{array}{|ll|ll|ll|}
4 & 2 & 2 & 6 & 3 & 2 \\
1 & 1 & 4 & 6 & 3 & 4 \\
4 & 5 & 6 & 5 & 5 & 3 \\
5 & 2 & 3 & 1 & 1 & 6 \\
\hline
\end{array} \\
& c_{8}^{5}=\begin{array}{|ll|ll|ll|}
5 & 3 & 6 & 2 & 4 & 6 \\
3 & 6 & 5 & 2 & 5 & 4 \\
4 & 1 & 1 & 1 & 2 & 4 \\
2 & 3 & 5 & 6 & 3 & 1 \\
\hline
\end{array} \\
& c_{8}^{8}=\begin{array}{|ll|ll|ll|}
6 & 6 & 4 & 1 & 4 & 5 \\
3 & 4 & 3 & 1 & 3 & 5 \\
4 & 2 & 2 & 1 & 2 & 5 \\
2 & 3 & 6 & 5 & 6 & 1 \\
\hline
\end{array} \\
& c_{8}^{11}=\begin{array}{|ll|ll|ll|}
2 & 4 & 1 & 5 & 3 & 4 \\
1 & 1 & 3 & 6 & 3 & 2 \\
3 & 6 & 5 & 5 & 6 & 2 \\
5 & 1 & 2 & 4 & 4 & 6 \\
\hline
\end{array}
\end{aligned}
$$

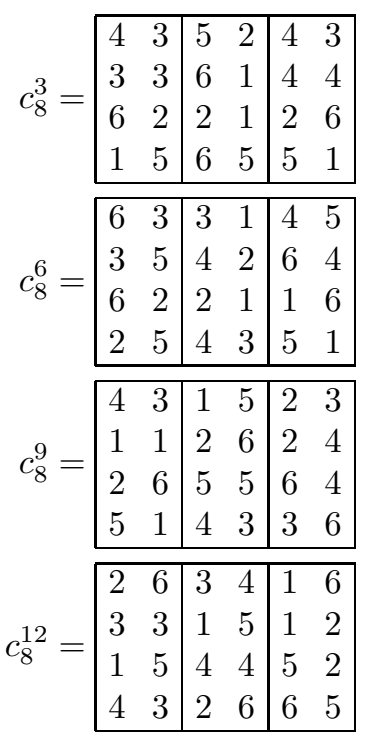


$\Delta_{9}:$

$$
\begin{aligned}
& c_{9}^{1}=\begin{array}{ll|ll|ll|}
5 & 4 & 3 & 2 & 5 & 3 \\
3 & 4 & 5 & 2 & 6 & 6 \\
6 & 2 & 1 & 2 & 1 & 5 \\
1 & 4 & 6 & 4 & 3 & 1 \\
\hline
\end{array} \\
& c_{9}^{2}=\begin{array}{ll|ll|ll|}
5 & 4 & 4 & 1 & 6 & 4 \\
3 & 3 & 5 & 1 & 6 & 5 \\
5 & 2 & 1 & 2 & 2 & 6 \\
2 & 4 & 6 & 3 & 3 & 1 \\
\hline
\end{array} \\
& c_{9}^{4}=\begin{array}{ll|ll|ll|}
6 & 4 & 4 & 1 & 6 & 3 \\
3 & 4 & 5 & 2 & 5 & 5 \\
6 & 1 & 2 & 2 & 2 & 5 \\
1 & 3 & 6 & 3 & 4 & 1 \\
\hline
\end{array} \\
& c_{9}^{5}=\begin{array}{ll|ll|ll|}
6 & 3 & 4 & 2 & 5 & 4 \\
3 & 4 & 6 & 2 & 6 & 5 \\
5 & 1 & 1 & 1 & 2 & 5 \\
2 & 3 & 6 & 4 & 3 & 1 \\
\hline
\end{array} \\
& c_{9}^{7}=\begin{array}{ll|ll|ll|}
5 & 6 & 3 & 2 & 4 & 6 \\
3 & 3 & 4 & 1 & 4 & 5 \\
4 & 1 & 2 & 2 & 1 & 5 \\
2 & 3 & 5 & 6 & 6 & 1 \\
\hline
\end{array} \\
& c_{9}^{8}=\begin{array}{ll|ll|ll|}
4 & 5 & 3 & 2 & 6 & 5 \\
3 & 3 & 6 & 1 & 6 & 4 \\
6 & 1 & 2 & 2 & 1 & 4 \\
2 & 3 & 4 & 5 & 5 & 1 \\
\hline
\end{array} \\
& c_{9}^{3}=\begin{array}{ll|ll|ll|}
6 & 3 & 4 & 2 & 6 & 3 \\
3 & 3 & 5 & 1 & 6 & 6 \\
5 & 2 & 2 & 1 & 2 & 5 \\
1 & 4 & 5 & 4 & 4 & 1 \\
\hline
\end{array} \\
& c_{9}^{6}=\begin{array}{|ll|ll|ll|}
5 & 3 & 3 & 1 & 6 & 4 \\
3 & 4 & 6 & 2 & 5 & 6 \\
5 & 2 & 2 & 1 & 1 & 5 \\
2 & 4 & 6 & 3 & 4 & 1 \\
\hline
\end{array} \\
& c_{9}^{9}=\begin{array}{lllll|ll|}
4 & 2 & 1 & 6 & 3 & 2 \\
1 & 1 & 3 & 5 & 3 & 4 \\
3 & 5 & 6 & 6 & 5 & 4 \\
6 & 1 & 4 & 2 & 2 & 5 \\
\hline
\end{array} \\
& c_{9}^{10}=\begin{array}{ll|ll|ll|}
2 & 4 & 3 & 5 & 1 & 4 \\
3 & 3 & 1 & 6 & 1 & 2 \\
1 & 6 & 5 & 5 & 6 & 2 \\
5 & 3 & 2 & 4 & 4 & 6 \\
\hline
\end{array}
\end{aligned}
$$

$\Delta_{10}$

$$
c_{10}=\begin{array}{ll|ll|ll}
6 & 4 & 3 & 2 & 5 & 4 \\
3 & 3 & 5 & 1 & 5 & 6 \\
5 & 1 & 2 & 2 & 1 & 6 \\
2 & 3 & 6 & 4 & 4 & 1 \\
\hline
\end{array}
$$

\section{Appendix B.}

On the next page we draw the induced subgraph of $\overline{\left\{c_{0}, c_{10}\right\}}$, together with the $i$-adjacency information. Recall that two adjacent chamber $c^{\prime}$ and $c^{\prime \prime}$ are said to be $i$-adjacent if $\tau\left(c^{\prime}\right)=\{i\} \cup \tau\left(c^{\prime} \cap c^{\prime \prime}\right)=\tau\left(c^{\prime \prime}\right)$. So 0-, 1-, 2-adjacency means that $c^{\prime}$ and $c^{\prime \prime}$ 'differ' only in, respectively, an octad, a trio, a sextet.

As mentioned earlier we only need describe $\left\{c_{0}\right\} \cup \Delta_{1} \cup \Delta_{2} \cup \Delta_{3} \cup \Delta_{4} \cup \Delta_{5}-\overline{\left\{c_{0}, c_{10}\right\}}$ can then be constructed from this using $y$.

So as not to clutter up our picture, the names of the chambers have been suppressed, with the exception of $c_{0}$ and $c_{j}^{1}(1 \leqslant j \leqslant 5)$. Reading downwards, in order, from $c_{j}^{1}$ gives the chambers $c_{j}^{k}$ in $\Delta_{j}$. Since $c_{0}, c_{1}^{1}, c_{1}^{2}, c_{1}^{3}, c_{1}^{4}, c_{1}^{5}$ and $c_{1}^{6}$ have the same octad and trio, any two of them are 2-adjacent. On our picture we have not drawn the 2 -adjacency between $c_{1}^{m}$ and $c_{1}^{n}(1 \leqslant m<n \leqslant 6)$. All other adjacencies within $\left\{c_{0}\right\} \cup \Delta_{1} \cup \Delta_{2} \cup \Delta_{3} \cup \Delta_{4} \cup \Delta_{5}$ are given. 


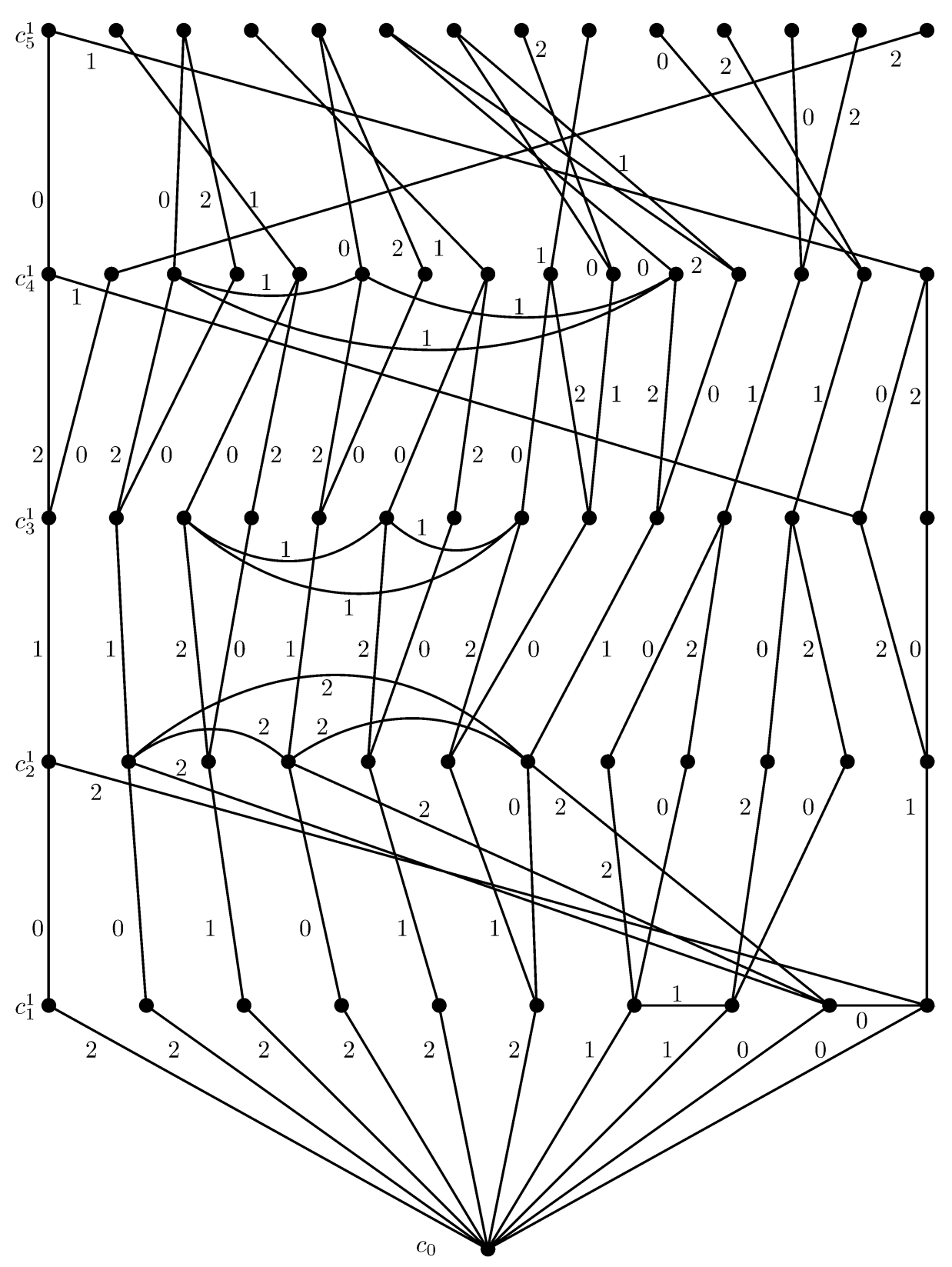


Appendix C.

$\mathrm{m} 24:=\operatorname{sub}<\operatorname{Sym}(24) \mid(1,2,3,4,5,6,7,8,9,10,11,12,13,14,15,16,17,18,19$, $20,21,22,23),(24,23)(3,19)(6,15)(9,5)(11,1)(4,20)(16,14)(13,21)>$; \#24 ;

244823040

sextet: $=$ sub<m24|Stabilizer $(\mathrm{m} 24,\{24,3,6,9\}), \mathrm{m} 24.2>$;

Index (m24, sextet);

1771

triopw: =Stabilizer (Stabilizer $(\mathrm{m} 24,\{22,18,8,12,2,10,17,7\})$,

$\{24,3,6,9,11,4,16,13\})$;

flag:=sub<m24|triopw,m24.2> meet sextet;

Index (m24,flag);

79695

CT : =CosetTable (m24,flag);

flagp:=CosetTableToPermutationGroup (m24,CT) ;

oo:=Orbits $($ Stabilizer $(\mathrm{flagp}, 1))$;

[\#०o[i]:i in [1..106]];

$[1,2,2,4,4,6,8,8,12,12,12,16,24,24,24,24,24,48$, $48,48,48,48,48,48,48,96,96,96,96,96,96,96,96,96$, $96,192,192,192,192,192,192,192,192,192,192,192,192$, $192,384,384,384,384,384,384,384,384,384,384,384,384$, $384,384,384,768,768,768,768,768,768,768,768,768,768$, $768,768,768,768,768,1536,1536,1536,1536,1536,1536$, $1536,1536,1536,1536,1536,1536,1536,1536,1536,1536,1536$, $1536,3072,3072,3072,3072,3072,3072,3072,3072,3072,3072$ ]

\section{References}

1. F. Buekenhout, 'The basic diagram of a geometry', Geometries and groups (Berlin, 1981), Lecture Notes in Mathematics 893 (Springer, Berlin-New York, 1981) 1-29. 120

2. J. H. Conway, R. T. Curtis, S. P. Norton, R. A. Parker and R. A. Wilson, Atlas of finite groups. Maximal subgroups and ordinary characters for simple groups. With computational assistance from J. G. Thackray (Oxford University Press, Eynsham, 1985). 123

3. R. T. Curtis, 'On the Mathieu group $M_{24}$ and related topics', PhD Thesis, Cambridge, 1972. 124

4. R. T. Curtis, 'A new combinatorial approach to $M_{24}$ ', Math. Proc. Cambridge Philos. Soc. 79 (1976) 25-42. 121, 122

5. R. T. Curtis, 'Eight octads suffice', J. Combin. Theory Ser. A 36 (1984) 116-123. 135

6. M. A. Ronan and S. D. Smith, '2-local geometries for some sporadic groups', Proc. Symp. Pure Math. 37 (1980) 283-289. 120, 121

7. M. Ronan, Lectures on buildings (Academic Press, Boston, MA, 1989). 120, 121 
8. P. Rowley, 'Chamber graphs of some sporadic group geometries', The atlas of finite groups ten years on (ed. R. T. Curtis and R. A. Wilson; Cambridge University Press, Cambridge, 1998) 249-260. 121, 122

9. J. Tits, 'A local approach to buildings', The geometric vein (ed. C. Davis, B. Grünbaum, F. A. Sherk; Springer, New York-Berlin, 1981) 519-547. 120

Peter Rowley

School of Mathematics

University of Manchester

Oxford Road

Manchester M13 9JL

United Kingdom 